\title{
FLORA DEL BOSQUE MESÓFILO DE MONTAÑA Y VEGETACIÓN adyacente en Avándaro, Valle de Bravo, eSTADO DE MÉXICO, MÉXICO
}

\author{
Yolanda López-Pérez', J. Daniel Tejero-DízZ1,3, Alin N. Torres-Díaz' e Isolda Luna-Vega² \\ ${ }^{1}$ Facultad de Estudios Superiores Iztacala, Universidad Nacional Autónoma de México \\ ${ }^{2}$ Facultad de Ciencias, Universidad Nacional Autónoma de México \\ ${ }^{3}$ Autor de correspondencia: tejero@servidor.unam.mx
}

\begin{abstract}
Resumen: El bosque mesófilo de montaña estudiado se encuentra en la zona central de la frontera entre la Faja Volcánica Transmexicana y la cuenca del río Balsas. En este trabajo se incluye una lista florística que comprende 98 familias, 245 géneros y 391 especies de plantas vasculares. La vegetación arbórea presenta tres estratos, uno alto (cercano a 40 m), donde son frecuentes varias especies de encinos y Zinowiewia integerrima; uno medio $(20 \mathrm{~m})$ con Carpinus caroliniana, Ternstroemia lineata subsp. lineata y Tilia americana, entre otras y uno bajo (5-15 m) con Clethra spp., Cleyera integrifolia, Cornus disciflora, Garrya laurifolia, Meliosma dentata, Oreopanax xalapensis, Parathesis melanosticta, Styrax argenteus y Symplocos citrea. Los estratos arbustivo y herbáceo son ricos debido a la intrusión de especies de zonas de uso agropecuario y recreativo. Se discute el estado de conservación del bosque con base en indicadores ambientales derivados de los resultados florísticos.
\end{abstract}

Palabras clave: biogeografía, tipos de vegetación, flora mesoamericana.

\begin{abstract}
The cloud forest studied in this work is located at the central part of the border between the Mexican Transvolcanic Belt and the Balsas basin. The floristic list of the area includes 98 families, 245 genera and 391 species of vascular plants. The arboreal vegetation is divided into three strata, a higher $(40 \mathrm{~m})$ mostly constituted by oaks and Zinowiewia integerrima, a medium (20 m) by Carpinus caroliniana, Ternstroemia lineata subsp. lineata and Tilia americana, among others, and a lower one (5-15 m) by Clethra spp., Cleyera integrifolia, Cornus disciflora, Garrya laurifolia, Meliosma dentata, Oreopanax xalapensis, Parathesis melanosticta, Styrax argenteus and Symplocos citrea. Shrubby and herbaceous layers are rich because the disturbance caused by the surrounding agricultural and recreational areas. We discussed the conservation status of the cloud forest based on environmental indicators derived from the floristic results.
\end{abstract}

Key words: biogeography, vegetation types, Mesoamerican flora.

$\mathbf{E}^{1}$ bosque mesófilo de montaña (BMM) posee una biodiversidad por unidad de área superior al de cualquier otro tipo de vegetación en México; tiene una composición florística y una estructura características, resultado de la compleja historia biogeográfica donde se mezclan elementos propios de zonas holárticas y neotropicales (Challenger, 1998; Rzedowski, 1996; Rzedowski, 2006; Villaseñor, 2010). Aunado a los anteriores aspectos biológicos intrínsecos, este tipo de vegetación desempeña un importante papel en los servicios ecosistémicos, principalmente para la provisión de agua (Challenger, 1998; Bonfil y Madrid, 2006).

El BMM ocupa una superficie muy reducida y fragmentada en México; Rzedowski (2006) estima que ocupa del 0.5 al $0.87 \%$ del territorio mexicano. Sin embargo, en la última década, la deforestación ha reducido su cobertura al menos en un $50 \%$, por lo que varios investigadores consideran a este ecosistema como un "hábitat en peligro de extinción" (Churchill et al., 1995; Challenger, 1998; Luna et al., 2001). Por su parte, la CONABIO (2010), ha clasificado como bosques de calidad media y altamente amenazados, a los que se localizan en la región de la cuenca alta del río Cutzamala (centro-occidente del estado de México), zona donde se ubica el área de estudio.

En general, el BMM en México ha sido estudiado desde el punto de vista florístico, ecológico y biogeográfico por varios autores (véase Ponce-Vargas et al., 2006). Sin embargo, sólo media docena de trabajos se centran en los BMM de la zona fisiográfica de influencia para este estudio (Leavenworth, 1946; Miranda, 1947; Luna et al., 1989; FragosoRamírez, 1990; Miranda-Jimenez y González-Ortiz, 1993; 
Torres-Zúñiga y Tejero-Díez, 1998). Aunque la cuenca hidrológica de Valle de Bravo es considerada como un Área Natural Protegida (SEMARNAT, 2005), forma parte de la región terrestre prioritaria 109 (Arriaga et al., 2000) y cuenta con un plan para el manejo hidrológico (CONAGUA, 2005), pocos son los estudios botánicos locales existentes. Entre ellos, se cuenta con las visitas históricas de Martín Sessé y Castillo en 1792, las colectas que realizó George B. Hinton en el área de Temascaltepec entre 1931 y 1935 (Hinton y Rzedowski, 1972) y la de Paray en 1936 (en Lagman, 1964) y Eizi Matuda entre 1953 y 1972, como parte de la Comisión Botánica Exploradora del estado de México (Romero-Romero y Meave, 2007). Recientemente, la CONABIO (2002) realizó un análisis del cambio de vegetación y uso de suelo en esta localidad, donde reportó una cobertura para el BMM de 6.21 ha en el año 2000.

Dada la importancia florística y de servicios ecosistémicos del BMM en la región de Valle de Bravo y el poco conocimiento local existente, el objetivo de este estudio es el de documentar la flora del BMM de la localidad de Avándaro en Valle de Bravo, así como discutir, a partir de indicadores florísticos, algunos aspectos de su estado de conservación.

\section{Material y Métodos}

Área de estudio. Avándaro se localiza al poniente de la ciudad de Toluca, en el municipio de Valle de Bravo, estado de México. El área de muestreo cubre un total de 1,870 ha, que se concentran en cuatro polígonos o microcuencas que desembocan en la presa de Valle de Bravo y forman parte del río Temascaltepec, tal como Cerro Gordo, el Cerro La Escalerilla, Casas Viejas y la cañada Los Saucos, entre los $19^{\circ} 07^{\prime}$ y $19^{\circ} 10^{\prime}$ latitud norte y $100^{\circ} 05^{\prime}$ y $100^{\circ} 10^{\prime}$ longitud oeste, a una altitud entre los 2,000 y 2,620 m (INEGI, 1998) (Figura 1).

Fisiográficamente Valle de Bravo se encuentra en la porción suroeste de la Faja Volcánica Transmexicana, correspondiente a la subprovincia fisiográfica Mil Cumbres, que es el sistema de topoformas más importante de esta provincia, con lomeríos de colinas redondeadas y mesetas de basalto (SPP, 1981). La zona oriental de la cuenca de Valle de Bravo se formó en diferentes episodios volcánicos; entre el Mioceno medio y tardío y posteriormente entre el Pleistoceno medio y el Holoceno. En el área de estudio los principales cerros como El Gordo y La Escalerilla están constituidos por escudos volcánicos monogenéticos de roca ígnea extrusiva máfica a intermedia, cubiertas por depósitos piroclásticos como tobas. En los alrededores de la presa de Valle de Bravo se encuentran depósitos superficiales neogénicos (Gómez-Tuena et al., 2005; Ferrari et al., 2007).

Los suelos que prevalecen son de tipo andosol y acrisol órtico; el primero deriva de cenizas volcánicas, se distribuye en la zona montañosa y presenta dos subtipos: húmico y ócrito. El acrisol órtico se caracteriza por ser moderadamen-

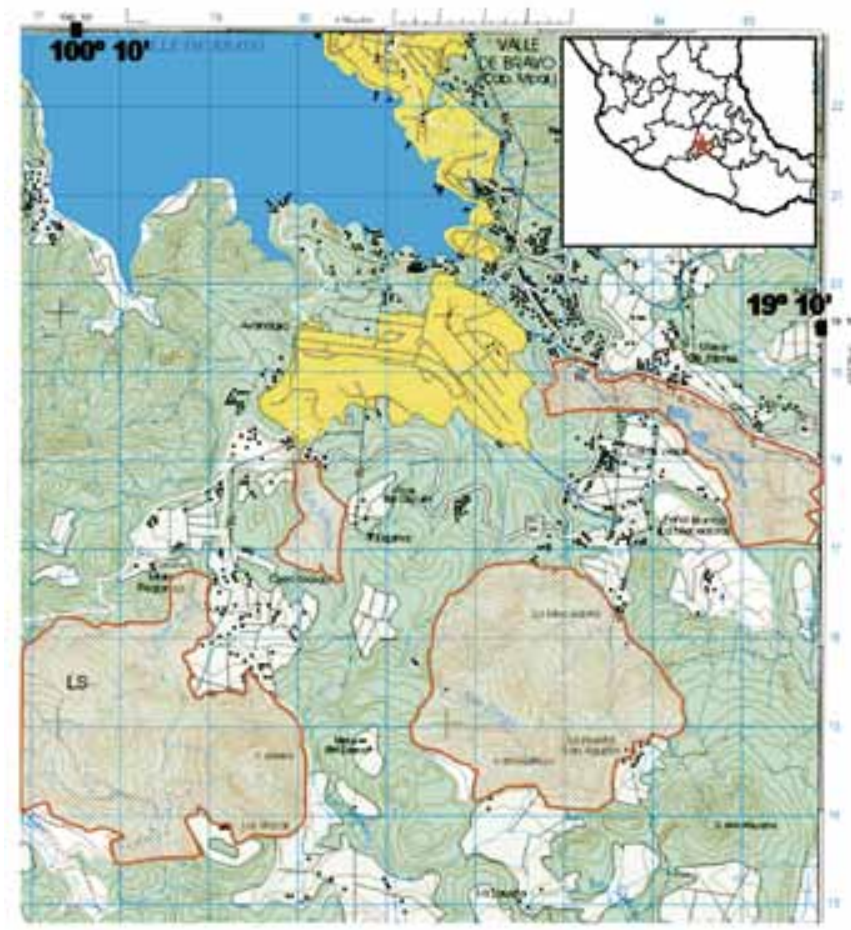

Figura 1. Ubicación del área de estudio (INEGI, 1998). Los polígonos amarillos corresponden a las zonas urbanas; los polígonos claros a las zonas agropecuarias; los polígonos rojos a las áreas estudiadas.

te susceptible a la erosión y se encuentra en las partes bajas y llanas fundamentalmente. Los leptosoles de roca ígnea afloran en forma de peñascos y taludes de muy poca extensión en las partes altas de los cerros Gordo y La Escalerilla (SPP, 1981).

De acuerdo a los datos de la estación meteorológica San Juan Guadalupe Hacienda, Valle de Bravo (19 $11^{\prime}$ N - $100^{\circ} 07^{\prime}$ O a 2,445 m s.n.m.), escogida por la cercanía geográfica y de altitud con el área de estudio, el clima es templado subhúmedo. Por el régimen de humedad es el más húmedo de los subhúmedos, con precipitación anual promedio de $1,001 \mathrm{~mm}$, la temporada de lluvias se presenta de mayo a octubre y los meses secos de noviembre a abril. La temperatura promedio anual es de $13.7^{\circ} \mathrm{C}$, el mes más cálido es mayo $\left(15.9^{\circ} \mathrm{C}\right)$ y el más frío enero $\left(11.4^{\circ} \mathrm{C}\right)$. Según García (2004), la fórmula climática correspondiente es $\mathrm{Cb}(\mathrm{w} 2)(\mathrm{w})(\mathrm{i}$ ')g (Figura 2). Las estaciones meteorológicas de Temascaltepec (1,800 m s.n.m.) y de Valle de Bravo (1,869 $\mathrm{m}$ s.n.m.), posicionadas de frente al sistema orográfico-convectivo de humedad que proviene de la cuenca del río Balsas (Jáuregui-Ostos y Vidal-Bello, 1981), indican que durante los meses de lluvias, unas cuantas horas del día pueden presentar neblinas, las cuales suman de 5 a 8 días al año.

El área de estudio contiene numerosos arroyos que nacen en los cerros de la porción sur de la cuenca de Valle de Bravo. El agua es almacenada en un sistema de tres presas 


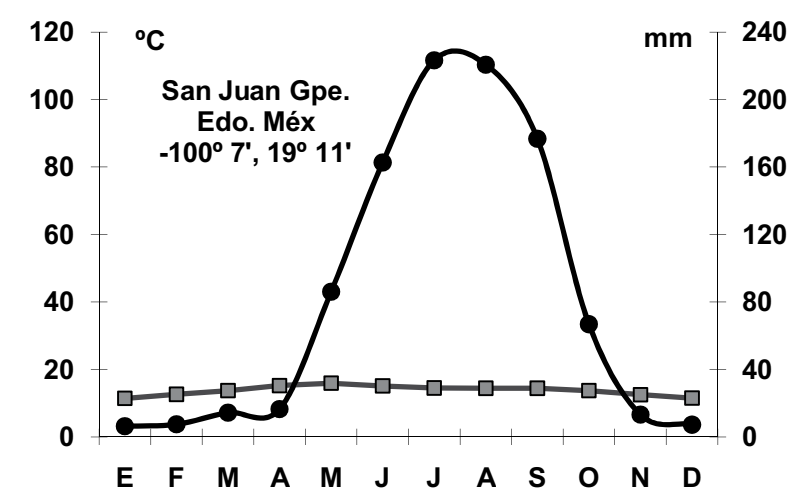

Figura 2. Diagrama ombrotérmico tipo Gaussen de la región de estudio obtenido a partir de la estación San Juan Guadalupe

(Valle de Bravo, Colorines y Santo Tomás), mismo que pertenece a la subcuenca del río Pungarancho-Temascaltepec, afluente del río Cutzamala y que desemboca, por el margen septentrional, al río Balsas. Este sistema de presas es el más importante almacén de agua en el área central de la Faja Volcánica Transmexicana y provee de servicios turísticos y parte importante de la electricidad y agua a las ciudades de Cuernavaca, Distrito Federal y Toluca (SPP, 1981).

Protocolo de muestreo. El trabajo de campo se realizó entre agosto de 1991 a diciembre de 1993 y de enero del 2003 a marzo del 2006. El material botánico se procesó mediante las técnicas convencionales para cada grupo taxonómico, de acuerdo a los criterios propuestos por Lot y Chiang (1986). En total se recolectaron cerca de 900 ejemplares, de los cuales se obtuvieron, además de los datos de geoposición, los siguientes:

a) Hábitat, de acuerdo al tipo de vegetación (Rzedowski,

2006): bosque mixto de Pinus - Quercus con elementos mesófilos, bosque mesófilo de montaña y vegetación secundaria derivada de la remoción de estos bosques.

b) Forma biológica según el sistema propuesto por Raunkiaer y modificado por Mueller-Dombois y Ellenberg (1974): terófito, criptófito, hemicriptófito, caméfito, fanerófito cespitoso, fanerófito escaposo, epífito, liana, parásita o hemiparásita e hidrófito.

El material botánico fue determinado mediante el uso de floras regionales y literatura especializada; el nombre fue corroborado mediante la comparación con material herborizado determinado por especialistas de los distintos grupos taxonómicos en los herbarios de la Facultad de Estudios Superiores Iztacala de la Universidad Nacional Autónoma de México (IZTA) y de la Escuela Nacional de Ciencias Biológicas del Instituto Politécnico Nacional (ENCB). Los ejemplares fueron depositados como referencia en los herbarios mencionados.

Para obtener indicadores ambientales se recopiló, para cada especie, la siguiente información a partir de la literatura: a) Flora sinantrópica: malezas, plantas silvestres que prosperan en ambientes antrópicos (Villaseñor-Ríos y Espinosa-García, 1998); introducidas, plantas no nativas de México de acuerdo a Villaseñor y Espinosa-García (2004) y de disturbio (recurrentes), especies que incrementan su población en claros y bordes de bosques (Rzedowski et al., 2001).

b) Distribución geográfica, obtenida a partir de las floras regionales y de la base de datos Tropicos.org, del Missouri Botanical Garden.

c) Especies que ameritan atención especial por ser raras, endémicas o estar dentro de alguna categoría de riesgo dentro de la Norma Oficial Mexicana-059 (SEMARNAT, 2010) y la Lista Roja de la IUCN (2010).

Los datos anteriores, se organizaron en una matriz de datos, donde el listado florístico siguió el esquema de Smith et al. (2006) para los pteridobiontes y de Calderón-de Rzedowski y Rzedowski (2001) para espermatobiontes.

Se utilizó el índice de biodiversidad taxonómica $(I B)$, definido por el número de especies dividido entre logaritmo natural del área en $\mathrm{km}^{2}(I B=S / \ln A$, donde $S$ será el número de especies registradas y $A$ el tamaño del área) para comparar la riqueza florística del BMM en Avándaro contra el de otros sitios con BMM en la República Mexicana (Squeo et al.,1998).

Finalmente, la caracterización y descripción del bosque se realizó tomando en cuenta las especies dominantes y su fisonomía. Con la finalidad de ilustrar la estructura de la vegetación, se llevó a cabo un diagrama del perfil del arbolado. Este se diseñó a partir de un rectángulo de $60 \times 8$ m que se colocó transversalmente a la cañada de Casas Viejas. Se utilizó como base un plano topográfico local de escala 1:200.

\section{Resultados}

Listado florístico. Se obtuvo un listado compuesto de 391 especies, que pertenecen a 245 géneros y 98 familias (ver Apéndice). De las familias encontradas, una pertenece a Lycopodiophyta, 11 a Polypodiophyta, una a Pinophyta y el resto a Magnoliophyta. Las familias representadas con $10 \mathrm{o}$ más especies son: Asteraceae (74 spp., 18.9\%), Poaceae (22, $5.6 \%)$, Fabaceae (21, 5.4\%), Lamiaceae (20, 5.1\%), Orchidaceae $(16,4.1 \%)$, Fagaceae y Solanaceae $(11,2.8 \%$ cada una). En estas siete familias se concentra aproximadamente el 48\% de las especies encontradas (Figura 3).

Composición florística. En la localidad de Avándaro, el BMM prospera principalmente en las laderas de orientación norte y aquellas cercanas a los arroyos. Algunos elementos higrófilos del BMM penetran al bosque mixto de Pinus-Quercus dado que ambos tipos de vegetación forman un gradiente con respecto a la humedad. La vegetación natural se encuentra fragmentada por terrenos que han sido utilizados para actividades agropecuarias o bien, zonas se- 


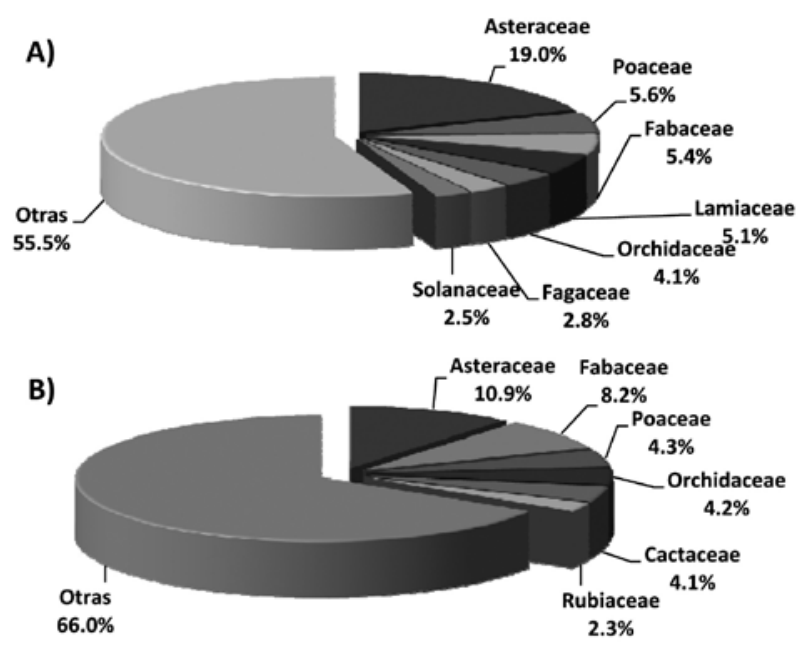

Figura 3. Familias mejor representadas: A) en Avándaro, Valle de Bravo, estado de México, B) a nivel nacional (Rzedowski, 1991).

miurbanas dotadas de elementos arquitectónicos y jardines (Figura 1).

El bosque de Pinus-Quercus aledaño al BMM está compuesto de dos estratos arbóreos; el primero, con altura entre 20 y $30 \mathrm{~m}$, está dominado por especies de Pinus ( $P$. douglasiana y $P$. teocote); el segundo, con una altura entre los 8 y $15 \mathrm{~m}$, está representado por varias especies de Quercus (principalmente $Q$. scytophylla) y elementos higrófilos muy separados entre sí y propios del BMM como Cleyera integrifolia, Oreopanax xalapensis, Styrax argenteus, Symplocos citrea y Ternstroemia lineata subsp. lineata. En los sitios conservados los estratos arbustivo y herbáceo son escasos, fundamentalmente debido a que el suelo se encuentra cubierto por una capa densa de hojarasca.

El BMM presenta tres estratos arbóreos; el superior, entre los 20 y $40 \mathrm{~m}$ de altura, puede presentar dos niveles y está compuesto por Carpinus caroliniana, Dendropanax arboreus, Quercus candicans, Q. laurina y Zinowiewia integerrima; en el estrato inferior, entre los 5 y $15 \mathrm{~m}$, dominan especies como Cornus disciflora, Clethra spp., Cleyera integrifolia, Oreopanax xalapensis, Styrax argenteus y Ternstroemia lineata subsp. lineata. Esporádicamente se pueden encontrar individuos de Dendropanax arboreus, Fraxinus uhdei, Garrya laurifolia y Meliosma dentata (Figura 4).

El estrato arbustivo es rico en especies, pero éstas no llegan a ser abundantes en lugares conservados. Las especies más importantes son: Ageratina mairetiana, Archibaccharis serratifolia, Astragalus strigulosus, Monnina ciliolata, Parathesis melanosticta, Roldana spp. y Salvia fulgens. El estrato herbáceo está pobremente representado y en la parte más húmeda predominan Adiantum andicola, Asplenium monanthes, Begonia spp., Cuphea spp., Lepechinia nelsonii, Polypodium subpetiolatum, Salvia spp. y Zeugites americana. El grupo de las epífitas está representado prin- cipalmente por Asplenium praemorsum, Epidendrum anisatum, Pecluma alfredii, Peperomia spp., Pleopeltis polylepis, Polypodium madrense, Tillandsia prodigiosa y Trichocentrum pachyphyllum. Entre las herbáceas y trepadoras más notorias, que generalmente se encuentran en sitios secundarios, se encuentran Clematis dioica, Cologania grandiflora, Gonolobus uniflorus, Ipomoea purpurea, Passiflora pavonis, Rubus liebmannii, Smilax pringlei y Solandra nitida. Las parásitas y hemiparásitas están presentes en los sitios más perturbados, principalmente Corallorhiza wisteriana, Phoradendron velutinum y Psittacanthus calyculatus.

Riqueza florística. El área de estudio presenta una riqueza florística de 133.51 especies $/ \mathrm{km}^{2}$. La comparación con respecto a otros BMM de México no es del todo exacta debido a varios aspectos: la complejidad del mosaico que forma el BMM y los límites difusos con respecto a la vegetación aledaña, el esfuerzo de muestreo diferencial entre los autores y la inexactitud en el cálculo de los límites del área de estudio. Aún con estas fuentes de errores, se estima que la riqueza florística de Avándaro es elevada (Cuadro 1).

Cuadro 1. Comparación de la riqueza florística de Avándaro, Valle de Bravo con otras áreas. Número de especies $=\mathrm{sp}$, logaritmo natural del área en $\mathrm{km}^{2}=\ln \mathrm{A}$. Sitios comparados: $1=\mathrm{BMM}$ en la sierra Madre Oriental. $2=$ BMM en la sierra Madre del Sur. 3= BMM en la Faja Volcánica Transmexicana/cuenca del Río Balsas.

\begin{tabular}{|c|c|c|c|c|}
\hline & $\begin{array}{l}\text { Altitud } \\
\text { (m s.n.m.) }\end{array}$ & $\begin{array}{l}\text { Área } \\
\left(\mathbf{k m}^{2}\right)\end{array}$ & $\begin{array}{l}\text { Número } \\
\text { total de es- } \\
\text { pecies (sp) }\end{array}$ & $\begin{array}{l}\text { Riqueza } \\
(\mathrm{sp} / \ln A)\end{array}$ \\
\hline $\begin{array}{l}\text { 'Teocelo, Ver. } \\
\text { (Luna et al., 1988) }\end{array}$ & $1,100-1,630$ & $\times 21$ & 277 & 90.98 \\
\hline $\begin{array}{l}\text { 30cuilan, Morelos y } \\
\text { Edo. de México } \\
\text { (Luna et al., 1989) }\end{array}$ & $1,800-2,400$ & 12.2 & 160 & 63.96 \\
\hline $\begin{array}{l}\text { ²Omiltemi, Gro. } \\
\text { (Jiménez-Ramírez } \\
\text { et al., 1993) }\end{array}$ & $2,300-2,700$ & $\times 30$ & 244 & 71.73 \\
\hline $\begin{array}{l}{ }^{2} \text { Miahuatlán, Oax. } \\
\text { (Campos-Villanueva y } \\
\text { Villaseñor, 1995) }\end{array}$ & $1,000-1,400$ & $\times 43$ & 353 & 93.85 \\
\hline $\begin{array}{l}{ }^{3} \text { Sierra de Sultepec, Edo. } \\
\text { de México (Torres-Zúñiga } \\
\text { y Tejero-Díez, 1998) }\end{array}$ & $1,500-2,800$ & 200 & 507 & 95.69 \\
\hline $\begin{array}{l}{ }^{1} \text { Landa de Matamoros, } \\
\text { Qro., Mex. (Cartujano } \\
\text { et al., 2002) }\end{array}$ & $800-2,200$ & $\times 54$ & 774 & 194.08 \\
\hline $\begin{array}{l}\text { Molote, Gro. } \\
\text { (Lozada et al, 2003) }\end{array}$ & $2,300-2,500$ & 7 & 458 & 235.37 \\
\hline $\begin{array}{l}\text { 'Lolotla, Hidalgo } \\
\text { (Ponce et al. 2006) }\end{array}$ & $1,050-1,580$ & 10.22 & 359 & 154.45 \\
\hline $\begin{array}{l}{ }^{3} \text { Valle de Bravo, Edo. } \\
\text { de México (este trabajo) }\end{array}$ & $2,000-2,620$ & 18.7 & 391 & 133.51 \\
\hline
\end{tabular}


Cuadro 2. Flora sinantrópica

\begin{tabular}{lcc}
\hline \multicolumn{1}{c}{ Comportamiento } & $\begin{array}{c}\mathbf{N}^{\mathbf{0}} \mathbf{d e} \\
\text { especies }\end{array}$ & $\mathbf{\%}$ \\
\hline Plantas de la vegetación no perturbada & $\mathbf{2 4 2}$ & $\mathbf{6 1 . 8 9}$ \\
$\begin{array}{l}\text { Plantas sinantrópicas } \\
\quad \text { Introducidas (Villaseñor y }\end{array}$ & $\mathbf{1 4 9}$ & $\mathbf{3 8 . 1 1}$ \\
$\quad \begin{array}{l}\text { Espinosa-García, 2004) } \\
\text { Malezas nativas (Villaseñor-Ríos y }\end{array}$ & 14 & 3.58 \\
$\quad \begin{array}{l}\text { Espinosa-García, 1998) } \\
\text { Plantas de disturbio (Calderón-de } \\
\text { Rzedowski y Rzedowski, 2001) }\end{array}$ & 25 & \\
\hline
\end{tabular}

Distribución geográfica de las especies. El $40.4 \%$ de las especies son de amplia distribución, es decir, rebasan los límites políticos y biológicos de México (Megaméxico 3 según Rzedowski, 1991); de éstas, la mayor parte se comparten con Sur y Centroamérica (19.9 y 9.71\% respectivamente). El 27.4\% del total se encuentra dentro de los límites biológicos de México (Megaméxico 3 según Rzedowski, 1991), entre ellas destacan las endémicas a Megaméxico 2 (Rzedowski, 1991) que representan el 17.7\%. El 32.2\% de la flora se restringe a los límites políticos de México, donde $10.5 \%$ es endémica a la provincia de la Faja Volcánica Transmexicana y a la cuenca del río Balsas (Cuadro 3). Ocho especies son endémicas locales, con una distribución restringida al estado de México y sus colindancias con Michoacán, Morelos o Distrito Federal (ver Apéndice).

Formas biológicas. Los hemicriptófitos son la forma de vida dominante con participación decreciente de fanerófi-

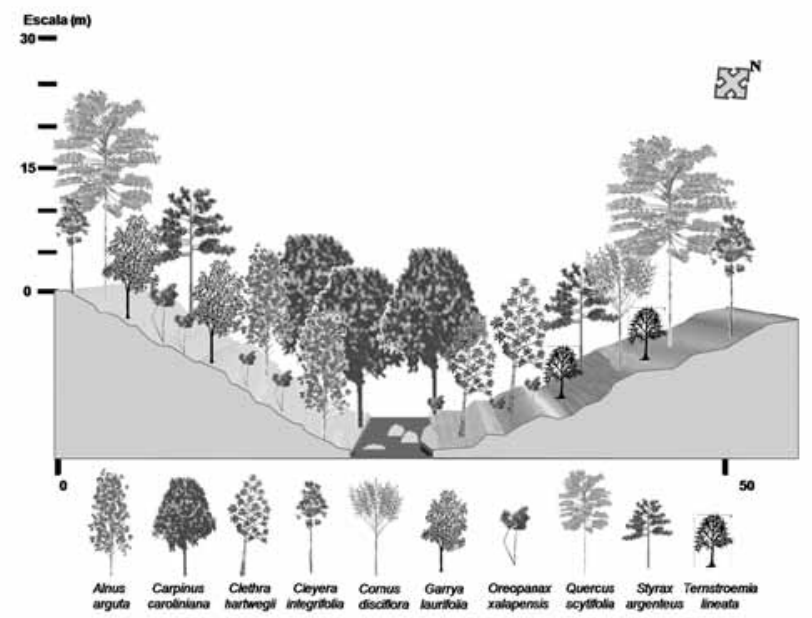

Figura 4. Diagrama de perfil topográfico con arbolado del bosque mesófilo de montaña en la Cuenca del río Amanalco en el predio de Casas Viejas, Avándaro, municipio Valle de Bravo. Coordenadas: $19^{\circ} 09^{\prime} 10^{\prime \prime} \mathrm{N}$ y $100^{\circ} 05^{\prime} 43$ 'O, altitud de 2,071 m s.n.m. Basado en un plano topográfico 1:200. Dibujó: D. Tejero-Díez, L. Rangel y C. Ledesma-Corral.

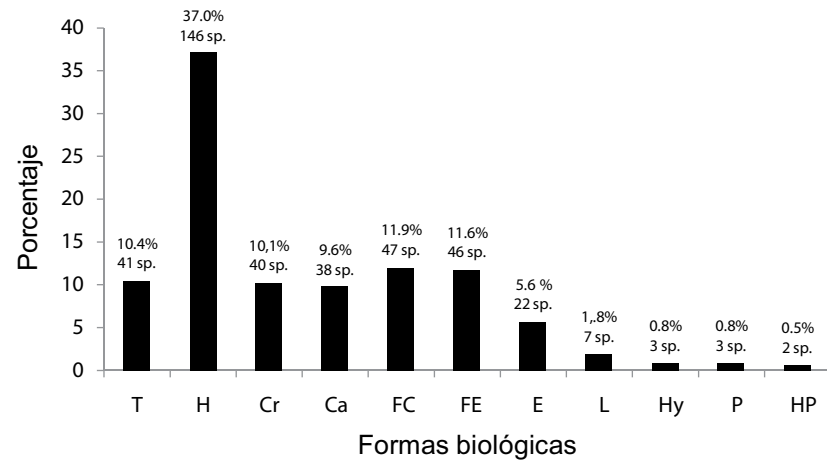

Figura 5. Espectro de formas biológicas en Avándaro, Valle de Bravo. $\mathrm{T}=$ terófito, $\mathrm{H}=$ hemicriptófito, $\mathrm{Cr}=$ criptófito, $\mathrm{Ca}=$ caméfito, $\mathrm{FC}=$ fanerófito cespitoso, $\mathrm{FE}=$ fanerófito escaposo, $\mathrm{E}=$ epífito, $\mathrm{L}=$ liana, $\mathrm{Hy}=$ hidrófito, $\mathrm{P}=$ parásita, $\mathrm{HP}=$ hemiparásita.

tos, criptófitos, caméfitos y epífitos. Las lianas, parásitas e hidrófitos son muy escasos, mientras que los terófitos tienen una representación similar a la de los fanerófitos (Figura 5).

Flora sinantrópica. Se puede estimar que cerca del $62 \%$ de las especies son propias de la vegetación natural y el 38\% restante son plantas indicadoras de las actividades humanas en la región. La mayoría de estas son clasificadas como malezas por Villaseñor-Ríos y Espinosa-García (1998) (Cuadro 2).

Especies que ameritan atención especial. Se encontraron cinco especies enlistadas en la NOM-059 (SEMARNAT, 2010) (dos en peligro de extinción, dos amenazadas y una con protección especial) y ocho en listados internacionales (IUCN, 2010, véase Apéndice). Se deben considerar también a las ocho especies endémicas locales mencionadas arriba.

\section{Discusión}

El conocimiento florístico del BMM de Avándaro en el municipio de Valle de Bravo es pobre, por lo que este trabajo representa un primer esfuerzo para obtener una lista florística del área y un diagnóstico ambiental en lo general.

La disposición en que se presentan las tres principales familias en la comunidad estudiada (Asteraceae, Fabaceae y Poaceae), es semejante al citado por Rzedowski (1991) para todo el país. El resto de las familias importantes en Avándaro se alejan del normal mexicano y están ligadas al tipo climático del área de estudio; Orchidaceae y Fagaceae son propias de la zona ecológica montañosa templada subhúmeda a húmeda del interior de la República. Fagaceae (especialmente el género Quercus) es cuantitativamente importante en el BMM (Rzedowski, 1996) y sobre todo en la Faja Volcánica Transmexicana (Miranda, 1947). En la zona de estudio, Orchidaceae ocupa el cuarto lugar (4.5\% de la 
Cuadro 3. Síntesis de la distribución de las especies presentes en Avándaro, Valle de Bravo (Rzedowski, 1991).

\begin{tabular}{cccc}
\hline Distribución & $\begin{array}{c}\text { Patrones de } \\
\text { distribución más } \\
\text { importantes }\end{array}$ & $\begin{array}{c}\text { No. de } \\
\text { especies }\end{array}$ & $\begin{array}{c}\text { Porcentaje } \\
\text { (respecto } \\
\text { al total) }\end{array}$ \\
\hline $\begin{array}{c}\text { Amplia distribución } \\
\text { 158 especies, 40.41\% }\end{array}$ & $\begin{array}{c}\text { México - Ámerica del Sur } \\
\text { México - Ámerica Central }\end{array}$ & 78 & 19.90 \\
Límites biológicos de & Megaméxico 1 & 20 & 9.71 \\
México 107 especies, & Megaméxico 2 & 69 & 17.65 \\
27.37\% & Megaméxico 3 & 18 & 4.60 \\
Límites políticos de & $\begin{array}{c}\text { Endémica a la Faja } \\
\text { México 126 especies, } \\
\text { 32.22\% }\end{array}$ & $\begin{array}{c}\text { Volcánica Transversal y } \\
\text { Depresión del Balsas } \\
\text { Endémica local }\end{array}$ & \\
& Endém & \\
& & & \\
&
\end{tabular}

flora), cifra inferior a lo que se reporta en otros sitios ubicados en las vertientes de barlovento de las sierras Madre Oriental y del Sur, lo que permite vislumbrar la menor humedad que caracteriza a los BMM de la sierra de Temascaltepec. La anterior afirmación se refuerza al comparar los datos de la estación meteorológica de Tlanchinol, Hgo., donde Orchidaceae representa el $7.5 \%$ de la flora (Ponce-Vargas et al., 2006); aquí se presentan 57 días de neblina y $2,324 \mathrm{~mm}$ de lluvia acumulada al año, a diferencia de los 5 a 8 días y $1,000 \mathrm{~mm}$ que se reportan en Avándaro.

El BMM de Avándaro se caracteriza por ser, como todos los bosques de la porción occidental de la Faja Volcánica Transmexicana, pobre en elementos higrófilos; están escasamente representadas o ausentes Araliaceae, Clusiaceae, Cyatheaceae, Juglandaceae, Lauraceae, Melastomataceae y Polypodiaceae, así como géneros propios de los BMM de la vertiente del Golfo de México como Drymis, Podocarpus y Liquidambar styraciflua. Por otro lado, están presentes algunos géneros de afinidades boreales como Carpinus, Cornus y Prunus totalmente ausentes en algunos BMM del sureste del país (Acosta-Castellanos, 1997). Estas diferencias en la composición florística, según el análisis fenético realizado por Acosta (2004), agrupan a los BMM de la Faja Volcánica Transmexicana en un conjunto aislado al resto de los BMM de México.

A su vez, el carácter templado subhúmedo del BMM de Avándaro limita el desarrollo de especies como Abies religiosa, Acer negundo, Cupressus spp., Ardisia compressa, Bocconia arborea, Ilex tolucana, Ostrya virginiana, Phoebe ehrenbergii, Phyllonoma laticuspis, Sambucus mexicana y Saurauia reticulata, entre otras, que están reportadas en sitios cercanos más fríos o más cálidos de la cuenca de México o de la propia cuenca del río Balsas por Miranda (1947), Rzedowski (1970), Luna et al. (1989) y Torres-Zúñiga y Tejero-Díez (1998). Sin embargo, la presencia en el BMM de Avándaro de géneros arbóreos propios del BMM de México (Rzedowski, 1996) como Alnus, Carpinus, Clethra, Cleye- ra, Cornus, Dendropanax, Fraxinus, Meliosma, Oreopanax, Styrax, Symplocos, Ternstroemia y Zinowiewia, permite diferenciar esta comunidad de los bosques de encino húmedo, que se encuentran en el mismo piso altitudinal, generalmente dominados por Quercus candicans y/o $Q$. scytophylla en la cuenca del río Balsas.

En conjunto, la flora en Avándaro muestra un patrón de distribución típico para los BMM de México y el porcentaje de endemismo a los límites políticos del país (32.2\%) es igual al pronosticado por Rzedowski (1991). Un amplio contingente se extiende hacia América Central y del Sur, mientras que los elementos norteños son escasos (Luna et al., 1989, 1994; Acosta-Castellanos, 1997).

Dado que las formas biológicas son la expresión adaptativa de las plantas a las presiones que ejerce el medio ambiente (Mueller-Dombois y Ellenberg, 1974; Krebs, 1985), entonces el espectro florístico proporciona datos interesantes de la historia biológica de la región. En el área de estudio, este espectro muestra semejanza al descrito como normal para los bosques de Pinus y de Quercus de la zona templada subhúmeda en México (Rzedowski, 2006), donde los hemicriptófitos son la forma de vida relevante. Sin embargo, los fanerófitos escaposos y epífitos tienen un porcentaje más elevado que en los bosques templados del mismo piso altitudinal probablemente favorecido por el carácter higrófilo en la zona de estudio. Por otro lado, los epífitos están mejor representados que en los bosques de la zona cálida, no así los fanerófitos escaposos (Challenger, 1998).

En cuanto al índice de biodiversidad taxonómica, el BMM de Avándaro presentó una riqueza elevada, comparada con otras zonas de la misma región biogeográfica, pero menor a la existente en los BMM del Golfo de México. Ello es producto de la menor humedad promedio en la parte occidental de la Faja Volcánica Transmexicana con respecto a la del Golfo de México (Velázquez et al., 2000) o la sierra Madre del Sur. A su vez, el carácter elevado de riqueza del BMM estudiado con respecto a otros bosques en la Faja Volcánica Transmexicana/cuenca del río Balsas probablemente se deba al grado de heterogeneidad del ambiente y a la gran cantidad de cauces fluviales. En términos de conservación regional, este aspecto es relevante dado que en un área pequeña se alberga una importante cantidad de especies, que incluye elementos endémicos, así como especies protegidas por normas nacionales e internacionales.

Aunque la vegetación y flora del BMM en el municipio de Valle de Bravo son relevantes en términos biológicos y de servicios ecosistémicos, este bosque ha sido severamente impactado; el mayor deterioro ha sido causado por el aumento de las zonas urbanas producto del desarrollo turístico, la tala a baja escala y las actividades agropecuarias (Bonfil y Madrid, 2006). El cambio de uso de suelo se ve reflejado en la existencia de un contingente importante de especies propias de zonas impactadas por actividad antrópica (38\% de las especies), v. gr. la sobrerrepresentación 
de las familias Lamiaceae y Solanaceae (Rzedowski, 1991; Calderón-de Rzedowski y Rzedowski, 2001) y la elevada presencia de la forma de vida terófita, propia de las zonas áridas y semiáridas, así como de hábitats abiertos temporalmente (Shimwell, 1971).

Por lo anterior, la zona de estudio tiene un estado de conservación bajo. Estos remanentes de BMM están fuertemente amenazados por el cambio de uso de suelo, lo que concuerda con el análisis realizado por la CONABIO (2010). El peligro potencial de este cambio de uso de suelo podría ser contraproducente para las propias actividades económicas de la región y de las grandes ciudades que son abastecidas de agua y electricidad (SEMARNAT, 2005; Bonfil y Madrid, 2006). Sin embargo, los remanentes de BMM y vegetación acompañante pueden recuperarse y conservarse con un manejo apropiado.

\section{Agradecimientos}

Parte de este trabajo fue la tesis de licenciatura de la primera autora. Se agradece a los curadores de los herbario IZTA y ENCB, Carlos Rojas Zenteno y Luz María Arreguín-Sánchez respectivamente, por permitir el acceso a sus colecciones. Ernesto Aguirre León auxilió en la determinación de orquídeas y Silvia Romero Rangel revisó la determinación de los encinos. Katia Juárez nos auxilió en la actualización de la lista florística. Luis Rangel y Canek Ledesma Corral ayudaron en la formación del diagrama de perfil.

\section{Literatura citada}

Acosta-Castellanos S. 1997. Afinidades fitogeográficas del bosque mesófilo de montaña de la zona de Pluma Hidalgo, Oaxaca, México. Polibotánica 6:25-39.

Acosta S. 2004. Afinidades de la flora genérica de algunos bosques mesófilos de montaña del nordeste, centro y sur de México: un enfoque fenético. Anales del Instituto de Biología, Serie Botánica 75:61-72.

Arriaga L., Espinoza J.M., Aguilar C., Martínez E., Gómez L. y Loa E. Coords. 2000. Regiones terrestres prioritarias de México. Escala de trabajo 1:1000000. Comisión Nacional para el Conocimiento y uso de la Biodiversidad. México. <www. conabio.gob.mx/conocimiento/regionalizacion/doctos/Tmapa. html > (Consultado 19 septiembre 2008).

Bonfil H. y Madrid L. 2006. El pago por servicios ambientales en la cuenca de Amanalco-Valle de Bravo. Gaceta Ecológica 80:63-79.

Calderón-de Rzedowski G. y Rzedowski J. 2001. Flora Fanerogámica del Valle de México. Instituto de Ecología A.C. y Comisión Nacional para el Conocimiento y Uso de la Biodiversidad, Pátzcuaro.

Campos-Villanueva A. y Villaseñor J.L. 1995. Estudio florístico de la porción central del municipio de San Jerónimo Coatlán, Distrito de Miahuatlán (Oaxaca). Boletín de la Sociedad Botánica de México 56:95-120.

Cartujano S., Zamudio S., Alcántara O. y Luna I. 2002. El bosque mesófilo de montaña en el municipio de Landa de Matamoros,
Querétaro, México. Boletín de la Sociedad Botánica de México 70:13-43.

Challenger A. 1998. Utilización y Conservación de los Ecosistemas Terrestres de México: Pasado, Presente y Futuro. Comisión Nacional para el Conocimiento y Uso de la Biodiversidad, Instituto de Biología, Universidad Nacional Autónoma de México y Agrupación Sierra Madre S.C., México, D.F.

Churchill S.P., Balslev H., Forero E. y Luteyn J.L. 1995. Biodiversity and conservation of Neotropical montane forests. Proceeding of the Neotropical Montane Forest. Biodiversity and Conservation Symposium, the New York Botanical Garden, 21 26 June 1993. The New York Botanical Garden, Nueva York.

CONABIO [Comisión Nacional para el Conocimiento y Uso de la Biodiversidad]. 2002. Monitoreo de ecosistemas. Caso Valle de Bravo. <www.conabio.gob.mx/conocimiento/cambios_ veg/doctos/cambios_valle.html> (Consultado 3 septiembre 2008).

CONABIO. 2010. El Bosque Mesófilo de Montaña en México: Amenazas y Oportunidades para su Conservación y Manejo Sostenible. Comisión Nacional para el Conocimiento y Uso de la Biodiversidad, México, D.F.

CONAGUA [Comisión Nacional del Agua]. 2005. Plan para la gestión integral del agua y recursos asociados de la Cuenca Valle de Bravo. <www.cuencaamanalcovalle.org/sec04_01.php > (Consultado 19 septiembre 2008).

Ferrari L., Rosas-Elguera J., Carrasco-Núñez G., Orozco-Esquivel M. y Norato-Cortez T. 2007. Cartografía geológica digital de la Faja Volcánica Trans-Mexicana y áreas adyacentes. Digital Geosciences, Universidad Nacional Autónoma de México. <digitalgeosciences.unam.mx/dgojs/index.php/DG/issue/ view/2-1>

Fragoso-Ramírez R. 1990. Estudio florístico en la parte alta de la Sierra de Zacualpan, Edo. de México. Tesis de licenciatura. Escuela Nacional de Estudios Profesionales Iztacala. Universidad Nacional Autónoma de México. Tlalnepantla, estado de México. $78 \mathrm{pp}$.

García E., 2004. Modificaciones al Sistema de Clasificación Climática de Köppen. Instituto de Geografía, Universidad Nacional Autónoma de México, México, D.F.

Gómez-Tuena A., Orozco-Esquivel M.T. y Ferrari L. 2005. Petrogénesis ígnea de la Faja Volcánica Transmexicana. Boletín de la Sociedad Geológica Mexicana 57:227-283.

Hinton J. y Rzedowski J. 1972. George B. Hinton, Collection of plants in southwestern México. Journal of the Arnold Arboretum 53:141-181

INEGI [Instituto Nacional de Estadística Geografía e Informática]. 1998. Carta topográfica. Valle de Bravo. E14A46. Escala 1: 50000 .

IUCN [International Union for Conservation of Nature and Natural Resources]. 2010. Red List of Threatened Species <www. iucnredlist.org > (Consultado febrero 2008).

Jáuregui-Ostos E. y Vidal-Bello J. 1981. Aspectos de la climatología del Estado de México. Boletín del Instituto de Geografía 11:21-54.

Jiménez-Ramírez J., Contreras-Jiménez J.L., González-Flores R.E., Antonio-Ocampo R., Lozano-Valdéz G. y Torres-Reynoso S. 1993. Plantas Vasculares. En: Luna I. y Llorente B.J. Eds. Historia Natural del Parque Ecológico Estatal Omiltemi, Chilpancingo, Guerrero, México, pp- 127-250, Universidad Nacional Autónoma de México, México, D.F. 
Krebs C.J., 1985. Ecología: Estudio de la Distribución y Abundancia. Harla, México, D.F.

Lagman I.K. 1964. A Selected Guide to the Literatura of the Flowering Plants of Mexico. University of Pennsylvania Press, Filadelfia.

Leavenworth W.C. 1946. A preliminary study of the vegetation of the region between Cerro Tancitaro and the Rio Tepacaltepec, Michoacan, Mexico. American Midland Naturalist 36:137-206.

Lot A. y Chiang F. (Compiladores). 1986. Manual de Herbario. Consejo Nacional de la Flora de México, A.C., México, D.F.

Lozada L., León M.E., Rojas J. y De Santiago R. 2003. Bosque mesófilo de montaña en el Molote. En: Diego-Pérez N. y Fonseca R.M. Eds. Estudios Florísticos en Guerrero. Fascículo No.13. Facultad de Ciencias. Universidad Nacional Autónoma de México, México, D.F.

Luna I., Almeida L., Villers L. y Lorenzo L. 1988. Reconocimiento florístico y consideraciones fitogeográficas del bosque mesófilo de montaña de Teocelo, Veracruz. Boletín de la Sociedad Botánica de México 48:35-63

Luna I., Almeida-Leñero L. y Llorente-Bousquets J. 1989. Florística y aspectos fitogeográficos del bosque mesófilo de montaña de las cañadas de Ocuilan, Estados de Morelos y México. Anales del Instituto de Biología, Serie Botánica 59:63-87.

Luna I., Ocegueda S. y Alcántara O. 1994. Florística y notas biogeográficas del bosque mesófilo de montaña del municipio de Tlanchinol, Hidalgo, México. Anales del Instituto de Biología, Serie Botánica 65:31-62.

Luna I., Velázquez A. y Velázquez E. 2001. El bosque mesófilo de México. En: Kappelle M. y Brown A.D. Eds. Bosques Nublados del Neotrópico, pp.183-229, Instituto Nacional de Biodiversidad (INBio), FUA, Unión Mundial para la Naturaleza (UICN), 2002 Año Internacional de las Montañas, Costa Rica.

Miranda F. 1947. Estudio sobre la vegetación de México V. Rasgos de la vegetación de la Cuenca del Río Balsas. Revista de la Sociedad Mexicana de Historia Natural 8:95-114.

Miranda-Jimenez M.A. y González-Ortiz M.A. 1993. Estudio de la vegetación y florística de la mesa basáltica de Holotepec, Distrito de Tenango del Valle, estado de México. Tesis de licenciatura. Escuela Nacional de Estudios Profesionales Iztacala. Universidad Nacional Autónoma de México. Tlalnepantla, estado de México. 74 pp.

Mueller-Dombois D. y Ellenberg H. 1974. Aims and Methods of Vegetation Ecology. John Wiley \& Sons, Nueva York.

Ponce-Vargas A., Luna-Vega I., Alcántara-Ayala O. y Ruiz-Jiménez C. 2006. Florística del bosque mesófilo de montaña de Monte Grande, Lolotla, Hidalgo, México. Revista Mexicana de Biodiversidad 77:177-190.

Romero-Romero M.A. y Meave J.A. 2007. Lista de contribuciones publicadas en el Boletín de la Sociedad Botánica de México durante el periodo enero de 1944 - junio de 2007. Boletín de la Sociedad Botánica de México 80: 113-138

Rzedowski J. 1970. Notas sobre el bosque mesófilo de montaña en el Valle de México. Anales de la Escuela Nacional de Ciencias Biológicas 18:91-106.

Rzedowski J. 1991. Diversidad y orígenes de la flora fanerogámica de México. Acta Botanica Mexicana 14:3-21.
Rzedowski J. 1996. Análisis preliminar de la flora vascular de los bosques mesófilos de montaña en México. Acta Botanica Mexicana 35:25-44.

Rzedowski J. 2006. Vegetación de México. Comisión Nacional para el Conocimiento y Uso de la Biodiversidad. México. <www.biodiversidad.gob.mx/publicaciones/librosDig/pdf/VegetacionMx_Cont.pdf>

SEMARNAT [Secretaría del Medio Ambiente y Recursos Naturales] 2005. Acuerdo por el que se determina como Zona Natural Protegida de competencia Federal, con la categoría de Área de Protección de Recursos Naturales Zona Protectora Forestal, los terrenos constitutivos de las cuencas de los ríos Valle de Bravo, Malacatepec, Tilostoc y Temascaltepec, estado de México. Diario Oficial de la Federación 1a Sección, 23 de junio de 2005. <www.semarnat.gob.mx/leyesynormas/SEMARNAT\%20 DOF/230605.1.pdf $>$ (Consultado 13 mayo 2010).

SEMARNAT. 2010. Norma Oficial Mexicana NOM-059-SEMARNAT-2010, Protección ambiental - Especies nativas de México de Flora y Fauna Silvestres - Categorías de Riesgo y especificaciones para su inclusión, exclusión o cambio - Lista de Especies en Riesgo. Diario Oficial de la Federación $2^{\text {a }}$ Sección, 30 de diciembre del 2010. <http://www.semarnat.gob.mx/ leyesynormas/SEMARNAT\%20DOF/Norma\%20Oficial\%20 Mexicana\%20NOM-059-SEMARNAT-2010.pdf>

Shimwell D.W. 1971. The Description and Classification of Vegetation. University of Washington Press, Seattle.

Smith A.R., Pryer K.M., Schuettpelz E., Korall P., Schneider H. y Wolf P.G. 2006. A classification for extant ferns. Taxon 55:705-731.

SPP [Secretaría de Programación y Presupuesto]. 1981. Síntesis de Información Geográfica del Estado de México. Coordinación General de los Servicios Nacionales de Estadística, Geografía e Informática, México, D.F.

Squeo F.A., Cavieres L.A., Arancio G., Novoa J.E., Matthei O., Marticorena C., Rodríguez R., Arroyo M.T.K. y Muñoz M. 1998. Biodiversidad vegetal de Antofagasta. Revista Chilena de Historia Natural 71:571-591.

Torres-Zúñiga M.M. y Tejero-Díez J.D. 1998. Flora y vegetación de la Sierra de Sultepec, estado de México. Anales del Instituto de Biología, Serie Botánica 69:135-174.

Tropicos.org. Missouri Botanical Garden. <www.tropicos.org> (Consultado 22 septiembre 2008).

Velázquez A., Toledo V.M. y Luna I. 2000. Mexican temperate vegetation. En: Barbour M.G. y Billings W.D. Eds. North American Terrestrial Vegetation, pp.573-592. Cambridge University Press, Cambridge.

Villaseñor-Ríos J.L. y Espinosa-García F.J. 1998. Catálogo de Malezas de México. Universidad Nacional Autónoma de México, Consejo Nacional Consultivo Fitosanitario y Fondo de Cultura Económica, México, D.F.

Villaseñor J.L. y Espinosa-García F.J. 2004. The alien flowering plants of Mexico. Diversity and Distributions 10:113-123.

Villaseñor J.L. 2010. El Bosque Húmedo de Montaña en México y sus Plantas Vasculares: Catálogo Florístico-Taxonómico. Comisión Nacional para el Conocimiento y Uso de la Biodiversidad y Universidad Nacional Autónoma de México, México, D.F

Recibido: 26 de mayo del 2010

Aceptado: 18 de abril de 2011 
Apéndice: Listado florístico Columnas: $\mathbf{F B}=$ Forma biológica $\mathrm{T}=$ terófito, $\mathrm{H}=$ hemicriptófito, $\mathrm{Cr}=$ criptófito, $\mathrm{Ca}=$ caméfito, $\mathrm{E}=$ epífito, $\mathrm{FC}=$ fanerófito cespitoso, $\mathrm{FE}=$ fanerófito escaposo, $\mathrm{L}=$ liana, $\mathrm{HP}=$ hemiparásita, $\mathrm{P}=$ parásita, $\mathrm{Hi}=$ hidrófita. Vegetación: $\mathrm{BMM}=$ bosque mesófilo de montaña; $\mathrm{BPQ}=$ bosque de Pinus-Quercus; $\mathrm{VS}$ y $\mathrm{PI}=$ Vegetación secundaria y pastizal inducido. Distribución: $\mathrm{Cos}=$ cosmopolita; $\mathrm{A}=$ americana; $\mathrm{SA}=$ América del Sur; $\mathrm{CA}=$ América Central; $\mathrm{An}=$ Antillas; M3 = Megaméxico 3; M2 = Megaméxico 2; M1 = Megaméxico 1; M = México (sensu Rzedowski, 1991); E-EVT = endémica a la Faja Volcánica Transmexicana; E-DB = endémica a la Cuenca del Río Balsas; E-SMOc = endémica a la Sierra Madre Occidental; $\mathrm{E}-\mathrm{CP}=$ endémica a la costa del Pacífico; $\mathrm{E}-\mathrm{I}=$ endémica local. Flora sinantrópica: mal = maleza, int $=$ introducida, dis $=$ disturbio. Categoría de riesgo: 1 = según la NOM-059 (SEMARNAT, 2010); $\mathrm{P}=$ peligro de extinción; $\mathrm{A}=$ amenazada $(*$ = endémica); $\mathrm{Pr}=$ Protección especial; 2 = según IUCN (2010); Br = bajo riesgo; $\mathrm{V}=$ vulnerable.

\begin{tabular}{lcccc}
\hline No. Familia/ especie & FB & Vegetación Distribución & $\begin{array}{c}\text { Flora } \\
\text { sinantrópica }\end{array} \begin{array}{c}\text { Categoría } \\
\text { de riesgo }\end{array}$ \\
\hline
\end{tabular}

\section{LICOPODIOPHYTA}

\section{Selaginellaceae}

1 Selaginella pallescens (C.Presl) Spring

2 Selaginella porphyrospora A.Braun

$\begin{array}{lll}\mathrm{Ca} & \mathrm{BMM} & \mathrm{M}-\mathrm{SA} \\ \mathrm{Ca} & \mathrm{BMM} & \mathrm{M}-\mathrm{SA}\end{array}$

1: $P$

\section{POLYPODIOPHYTA}

\section{Aspleniaceae}

3 Asplenium cuspidatum Lam.

$4 \quad$ Asplenium hallbergii Mickel \& Beitel

5 Asplenium monanthes $\mathrm{L}$.

6 Asplenium praemorsum Sw.

\section{Blechnaceae}

7 Blechnum appendiculatum Willd.

8 Woodwardia spinulosa M.Martens \& Galeotti

\section{Dennstaedtiaceae}

9 Dennstaedtia distenta (Kunze) T.Moore

10 Pteridium aquilinum (L.) Kuhn var. feei (W.Schaffn. ex Fée) Maxon ex Yunck.

11 Pteridium arachnoideum (Kaulf.) Maxon

\section{Dryopteridaceae}

12 Ctenitis equestris (Kunze) Ching var. equestris

13 Dryopteris pseudofilix-mas (Fée) Rothm.

14 Elaphoglossum petiolatum (Sw.) Urb.

15 Polystichum distans E.Fourn.

16 Polystichum turrialbae Christ.

Lomariopsidaceae

17 Nephrolepis undulata (Afzel. ex Sw.) J.Sm.

Onocleaceae

18 Onocleopsis hintonii F.Ballard

Ophioglossaceae

19 Botrychium schaffneri Underw.

20 Botrychium virginianum (L.) Sw.

Polypodiaceae

21 Pecluma alfredii (Rosenst.) M.G.Price var. cupreolepis (A.M.Evans) A.R.Sm.

22 Pleopeltis polylepis (Roem. ex Kunze)

T.Moore var. polylepis

23 Polypodium furfuraceum Schltdl. \& Cham.

24 Polypodium madrense J.Sm.

25 Polypodium polypodioides (L.)

E

$\mathrm{H}$

$\mathrm{H}$

$H / E$

$\mathrm{H}$

$\mathrm{Ca}$

$\mathrm{Ca}$

$\mathrm{Cr}$

$\mathrm{Cr}$

$\mathrm{Cr}$

$\mathrm{Ca}$

$\mathrm{H}$

$\mathrm{Ca}$

$\mathrm{Ca}$

$\mathrm{H}$

$\mathrm{H}$

$\mathrm{Cr}$

$\mathrm{Cr}$

E

E

$E$

$\mathrm{E} / \mathrm{H}$

\section{BMM}

BMM, BPQ

BMM

BMM

BMM

BMM

BMM

BMM

BPQ

BMM

BMM

BMM

BMM

BMM

BMM

BMM

BMM

BMM

BMM

BMM

BPQ

BMM

BMM
M-SA

$M$

Cos

M-SA

M-SA

M2

M-SA

M3

Dis

M-An-SA

M-CA

M1

M-SA

M2

M-SA

Cos

Int

M2

M-SA

Cos

Dis

M-SA

M

M-SA

$M$

M-SA 
Apéndice: Continuación

No. Familia/ especie

26 Polypodium subpetiolatum Hook.

\section{Pteridaceae}

27 Adiantum andicola Liebm.

28 Adiantum braunii Mett. ex Kuhn

29 Adiantum poiretii Wikstr.

30 Bommeria pedata (Sw.) Fourn.

31 Cheilanthes arizonica (Maxon) Mickel

32 Cheilanthes farinosa (Forssk.) Kaulf.

33 Pityrogramma ebenea (L.) Proctor

34 Pteris cretica L.

Thelypteridaceae

35 Thelypteris oligocarpa (Humb. \& Bonpl. ex Willd.) Ching

36 Thelypteris pilosa (M.Martens \& Galeotti) Crawford

Thelypteris rudis (Kunze) Proctor

Woodsiaceae

38 Cystopteris fragilis (L.) Bernh.

39 Diplazium lonchophyllum Kunze

\section{PINOPHYTA}

\section{Pinaceae}

40 Pinus devoniana Lindl.

41 Pinus douglasiana Martínez

42 Pinus leiophylla Schltdl. \& Cham. var. leiophylla

43 Pinus montezumae Lamb.

44 Pinus pseudostrobus Lindl. var. pseudostrobus

45 Pinus teocote Schltdl. \& Cham

MAGNOLIOPHYTA

MAGNOLIOPSIDA

Amaranthaceae

\section{Anacardiaceae}

47 Toxicodendron radicans (L.) Kuntze

\section{Apiaceae}

Arracacia atropurpurea (Lehm.)

Benth. \& Hook.f. ex Hemsl.

49 Bowlesia flabilis J.F.Macbr.

50 Cyclospermum leptophyllum (Pers.) Sprague

51 Daucus montanus Humb. \& Bonpl. ex Spreng.

52 Donnellsmithia mexicana (B.L.Rob.)

Mathias \& Constance

53 Eryngium bonplandii F.Delaroche

54 Eryngium carlinae F.Delaroche

55 Eryngium subacaule Cav.

56 Sanicula liberta Cham. \& Schltdl.

\section{Araliaceae}

57 Dendropanax arboreus (L.) Decne. \& Planch.

58
Oreopanax xalapensis (Kunth) Decne. \& Planch.
FB Vegetación Distribución

$\begin{array}{cc}\text { Flora } & \text { Categoría } \\ \text { sinantrópica } & \text { de riesgo }\end{array}$

E

\section{BMM}

M3

$\mathrm{H}$

$\mathrm{H}$

$\mathrm{H}$

$\mathrm{H}$

$\mathrm{H}$

$\mathrm{H}$

$\mathrm{H}$

$\mathrm{H}$

Ca BMM

\section{BMM}

$M-S A$

BMM

M-SA

BMM

Cos

BMM

M-CA

BMM, BPQ

M3

BMM Cos

\section{BMM}

M-SA

BMM

Cos

M-SA

$\mathrm{H}$

Ca

BMM

M3

$\mathrm{H}$

BMM

M-SA

$\mathrm{H}$

BMM

Cos

H BMM

M-SA

FE

FE

FE

FE

FE

FE

$\begin{array}{cc}\text { BMM } & \text { M2 } \\ \text { BPQ } & \text { E-SMOcyDB } \\ \text { BMM, BPQ } & \text { M1 } \\ \text { BMM } & \text { M2 } \\ \text { BMM, BPQ } & \text { M2 } \\ \text { BMM } & M\end{array}$

2: $\mathrm{Br}$

2: $\mathrm{Br}$

2: $\mathrm{Br}$

2: $\mathrm{Br}$

2: $\mathrm{Br}$

2: $\mathrm{Br}$ 
Apéndice: Continuación

\section{No. Familia/ especie}

\section{FB Vegetación Distribución}

Flora Categoría sinantrópica de riesgo

\begin{tabular}{|c|c|c|c|c|c|}
\hline & Asclepiadaceae & & & & \\
\hline 59 & Asclepias angustifolia Schweigg. & $\mathrm{Ca}$ & $\mathrm{BMM}, \mathrm{BPQ}$ & M1 & \\
\hline \multirow[t]{2}{*}{60} & Gonolobus uniflorus Kunth & $\mathrm{L}$ & $\mathrm{BMM}$ & E-EVT & \\
\hline & Asteraceae & & & & \\
\hline 61 & Acourtia turbinata Reveal \& R.M. King & $\mathrm{Cr}$ & BMM & E-SMOcyDB & \\
\hline 62 & Ageratina areolaris (DC.) Gage ex B.L.Turner & FC & $\mathrm{BMM}$ & M-CA & \\
\hline 63 & Ageratina mairetiana (DC.) R.M.King \& H.Rob. & FC & BMM & $\mathrm{M}-\mathrm{CA}$ & \\
\hline 64 & Ageratina pazcuarensis (Kunth) R.M.King \& H.Rob. & $\mathrm{H}$ & $\mathrm{BPQ}$ & M2 & \\
\hline 65 & $\begin{array}{l}\text { Ageratina petiolaris (Moc. \& Sessé ex DC.) } \\
\text { R.M.King \& H.Rob }\end{array}$ & $\mathrm{Ca}$ & BMM & M & Dis \\
\hline 66 & Ageratina pichinchensis (Kunth) R.M.King \& H.Rob. & FC & $\mathrm{BMM}$ & E-I & \\
\hline 67 & Ageratina scorodonioides (A.Gray) R.M.King \& H.Rob. & FC & BMM & M & \\
\hline 68 & Ageratum corymbosum Zuccagni & $\mathrm{Ca}$ & $\mathrm{BMM}, \mathrm{BPQ}$ & M2 & Mal \\
\hline 69 & Ambrosia psilostachya DC. & $\mathrm{H}$ & VS y PI & NA-M & Mal \\
\hline 70 & Archibaccharis asperifolia (Benth.) S.F.Blake & FC & BPQ & M2 & Mal \\
\hline 71 & Archibaccharis serratifolia (Kunth) S.F.Blake & FC & $\mathrm{BMM}, \mathrm{BPQ}$ & M2 & Dis \\
\hline 72 & Aster subulatus Michx. var. subulatus & $\mathrm{T}$ & $\mathrm{BMM}$ & M-SA & Mal \\
\hline 73 & Baccharis conferta Kunth & FC & $\mathrm{BMM}, \mathrm{BPQ}$ & M & Mal \\
\hline 74 & Baccharis multiflora Kunth var. multiflora & $\mathrm{Ca}$ & BMM & M2 & \\
\hline 75 & Bidens aequisquama (Fernald) Sherff var. aequisquama & $\mathrm{H}$ & $\mathrm{BMM}$ & E-DByEVT & \\
\hline 76 & Bidens ostruthioides (DC.) Sch.Bip. & $\mathrm{H}$ & BMM & $\mathrm{M}-\mathrm{CA}$ & \\
\hline 77 & Bidens serrulata (Poir.) Desf. & $\mathrm{T}$ & BMM & M & Mal \\
\hline 78 & Bidens triplinervia Kunth & $\mathrm{H}$ & BMM & M-SA & Mal \\
\hline 79 & Brickellia secundiflora (Lag.) A.Gray var. secundiflora & FC & BMM & M & Mal \\
\hline 80 & Brickellia tomentella A.Gray & FC & $\mathrm{BMM}, \mathrm{BPQ}$ & E-DByEVT & \\
\hline 81 & Cirsium acantholepis Petr. & $\mathrm{H}$ & VS y PI & E-EVT & Dis \\
\hline 82 & Cirsium ehrenbergii Sch.Bip. & $\mathrm{H}$ & BMM & E-SMOcyEVT & Dis \\
\hline 83 & Chromolaena odorata (L.) R.M.King \& H.Rob. & FC & BMM & M1 & \\
\hline 84 & Conyza coronopifolia Kunth & $\mathrm{T}$ & VS y PI & M-SA & Mal \\
\hline 85 & Cosmos bipinnatus Cav. & $\mathrm{T}$ & VS y PI & M1 & Mal \\
\hline 86 & Dahlia coccinea Cav. & $\mathrm{Cr}$ & BMM & M2 & \\
\hline 87 & Desmanthodium fruticosum Greenm. & $\mathrm{Ca}$ & BMM & E-CPyDB & \\
\hline 88 & Erigeron karvinskianus DC. & $\mathrm{H}$ & BMM & Cos & Mal \\
\hline 89 & Erigeron longipes DC. & $\mathrm{H}$ & BMM & M2 & Mal \\
\hline 90 & Heterotheca inuloides Cass. & $\mathrm{H}$ & BMM & $M$ & Mal \\
\hline 91 & Hieracium abscissum Less. & $\mathrm{H}$ & $\mathrm{BMM}$ & $\mathrm{M}-\mathrm{CA}$ & \\
\hline 92 & Jaegeria hirta (Lag.) Less. & $\mathrm{T}$ & BMM & M-SA & Mal \\
\hline 93 & Lagascea helianthifolia Kunth var. helianthifolia & FC & $\mathrm{BPQ}$ & M & \\
\hline 94 & Melampodium divaricatum (Rich.) DC. & $\mathrm{T}$ & BMM & M-SA & Mal \\
\hline 95 & Melampodium strigosum Stuessy & $\mathrm{T}$ & VS y PI & M1 & Mal \\
\hline 96 & Montanoa grandiflora DC. & $\mathrm{Ca}$ & BMM & E-EVT & Mal \\
\hline 97 & Packera sanguisorbae (DC.) C.Jeffrey & $\mathrm{H}$ & BPQ & M & \\
\hline 98 & Perymenium berlandieri DC. & $\mathrm{Ca}$ & $\mathrm{BMM}$ & E-EVT & Mal \\
\hline 99 & Perymenium subsquarrosum B.L.Rob. \& Greenm. & FC & BMM & E-SMOc & \\
\hline 100 & Piqueria trinervia Cav. & $\mathrm{H}$ & $\mathrm{BMM}$ & M-An-CA & Mal \\
\hline 101 & Psacalium peltatum (Kunth) Cass. & $\mathrm{Cr}$ & $\mathrm{BMM}$ & M & \\
\hline 102 & Pseudognaphalium canescens (DC.) Anderb. & $\mathrm{Ca}$ & BMM & M1 & \\
\hline 103 & Pseudognaphalium chartaceum (Greenm.) Anderb. & $\mathrm{T}$ & BMM & E-DByEVT & Dis \\
\hline 104 & Pseudognaphalium inornatum (DC.) Anderb. & $\mathrm{H}$ & BMM & M & Dis \\
\hline
\end{tabular}


Apéndice: Continuación

No. Familia/ especie

FB Vegetación Distribución

$\begin{array}{cc}\text { Flora } & \begin{array}{c}\text { Categoría } \\ \text { sinantrópica riesgo }\end{array}\end{array}$

105 Pseudognaphalium oxyphyllum (DC.) Kirp.

106 Pseudognaphalium purpurascens (DC.) Anderb.

$\mathrm{H}$

107 Pseudognaphalium semiamplexicaule (DC.) Anderb.

108 Pseudognaphalium viscosum (Kunth) Anderb.

109 Roldana angulifolia (DC.) H.Rob. \& Brettell

110 Roldana barba-johannis (DC.) H.Rob. \& Brettell

111 Roldana lobata La Llave

112 Roldana sessilifolia (Hook. \& Arn.) H.Rob. \& Brettell

113 Roldana suffulta (Greenm.) H.Rob. \& Brettell

114 Rumfordia floribunda DC. var. australis R.W.Sanders

115 Sigesbeckia jorullensis Kunth

116 Sonchus oleraceus $\mathrm{L}$.

117 Stevia incognita Grashoff

118 Stevia monardifolia Kunth

119 Stevia origanoides Kunth

120 Stevia ovata Willd. var. ovata

121 Stevia pilosa Lag.

122 Stevia porphyrea McVaugh

123 Stevia serrata Cav.

124 Stevia subpubescens Lag.

125 Stevia viscida Kunth

126 Tagetes filifolia Lag.

127 Tagetes foetidissima DC.

128 Tagetes lucida Cav.

129 Tagetes lunulata Ortega

130 Taraxacum officinale F.H.Wigg.

131 Tridax trilobata Hemsl.

132 Verbesina oncophora B.L.Rob. \& Seaton

133 Verbesina tetraptera (Ortega) A.Gray

134 Vernonia alamanii DC.

\section{Begoniaceae}

135 Begonia gracilis Kunth

136 Begonia hintoniana L.B.Sm. \& B.G.Schub.

137 Begonia manicata Cels

Berberidaceae

138 Berberis moranensis Schult. ex Schult.f.

\section{Betulaceae}

139 Alnus arguta (Schltdl.) Spach

140 Carpinus caroliniana Walter Boraginaceae

141 Lasiarrhenum trinervium (Lehm) B.L.Turner Cactaceae

142 Heliocereus elegantissimus Britton \& Rose Campanulaceae

143 Diastatea micrantha (Kunth) McVaugh

144 Lobelia fenestralis Cav.

145 Lobelia gruina Cav.

146 Lobelia laxiflora Kunth

147 Lobelia longicaulis Brandegee

\begin{tabular}{|c|c|c|c|}
\hline BMM, VS y PI & M3 & Dis & \\
\hline BMM & $M$ & & \\
\hline BMM & M2 & & \\
\hline VS y PI & M3 & Dis & \\
\hline BMM, BPQ & $M$ & & \\
\hline BMM & M2 & & \\
\hline $\mathrm{BPQ}$ & E-DByEVT & Dis & \\
\hline BMM & E-SMOcyEVT & Dis & \\
\hline BMM & E-EVT & & \\
\hline BMM & M & & \\
\hline VS y PI & M-SA & Mal & \\
\hline VS y PI & $\operatorname{Cos}$ & Int & \\
\hline BMM, BPQ & M-SA & Mal & \\
\hline BMM & M & & \\
\hline BPQ & $M$ & & \\
\hline VS y PI & $A$ & Mal & \\
\hline BMM & $M$ & & \\
\hline BMM & $M$ & & \\
\hline BPQ & M-SA & Mal & \\
\hline BMM & E-SMOcyDB & & \\
\hline $\mathrm{BMM}$ & M3 & Mal & \\
\hline VS y PI & M-SA & Mal & \\
\hline BMM & $\mathrm{M}-\mathrm{CA}$ & Mal & \\
\hline BPQ & M2 & Mal & \\
\hline BMM, VS y PI & $\mathrm{M}-\mathrm{CA}$ & Mal & \\
\hline BMM, VS y PI & Cos & Int & \\
\hline VS y PI & E-EVT & Mal & \\
\hline BMM & E-EVT & & \\
\hline BMM & M & Dis & \\
\hline BMM & M & Mal & \\
\hline BMM & M2 & & \\
\hline BMM & E-I & & \\
\hline BMM & M2 & & \\
\hline BMM & M & & \\
\hline BMM & M-CA & & 2: $\mathrm{Br}$ \\
\hline BMM & M3 & & 1: A \\
\hline BMM & E-DByEVT & & \\
\hline BMM & E-SMOcyDB & & \\
\hline BMM & M-SA & Mal & \\
\hline BMM & M1 & Mal & \\
\hline BMM & $M$ & Mal & \\
\hline BMM & M1 & Mal & \\
\hline BMM & M-CA & & \\
\hline
\end{tabular}


Apéndice: Continuación

No. Familia/ especie

148 Lobelia schmitzii E.Wimm.

\section{Caprifoliaceae}

149 Symphoricarpos microphyllus Kunth

\section{Caryophyllaceae}

150 Arenaria lanuginosa (Michx.) Rohrb.

151 Stellaria cuspidata Willd. ex Schltdl

\section{Celastraceae}

152 Celastrus pringlei Rose

153 Zinowiewia integerrima (Turcz.) Turcz.

Cistaceae

154 Helianthemum glomeratum (Lag.) Lag. ex DC. Clethraceae

155 Clethra hartwegii Britton

156 Clethra mexicana DC.

Convolvulaceae

157 Cuscuta umbellata Kunth

158 Dichondra sericea Sw.

159 Evolvulus alsinoides L.

160 Ipomoea purpurea (L.) Roth

\section{Cornaceae}

161 Cornus disciflora Moc. \& Sessé. ex DC.

162 Cornus excelsa Kunth

\section{Cucurbitaceae}

163 Cyclanthera integrifoliola Cogn.

164 Microsechium helleri (Peyr) Cogn.

165 Sicyos deppei G.Don

Ericaceae

166 Arbutus xalapensis Kunth

167 Comarostaphylis longifolia (Benth.) Klotzsch

168 Monotropa hypopithys L.

\section{Euphorbiaceae}

169 Euphorbia graminea Jacq.

\section{Fabaceae}

170 Astragalus guatemalensis Hemsl. var. brevidentatus (Hemsl.) Barneby

171 Astragalus micranthus Desv.

172 Astragalus nutallianus DC.

173 Astragalus oxyrhynchus Hemsl.

174 Astragalus strigulosus Kunth

175 Calliandra grandiflora (L'Hér.) Benth.

176 Cologania biloba (Lindley) G.Nicholson

177 Cologania broussonetii (Balb.) DC.

178 Cologania obovata Schltdl.

179 Desmodium amplifolium Hemsl.

180 Desmodium grahamii A.Gray

181 Indigofera thibaudiana DC.

182 Leucaena esculenta (DC.) Benth.

183 Lupinus campestris Schltdl. \& Cham.

184 Lupinus splendens Rose

\section{FB \\ Vegetación Distribución}

Flora Categoría sinantrópica de riesgo

$\begin{array}{cccc}\text { T } & \text { BMM } & \text { E-I } & \\ \text { FC } & \text { BMM } & \text { M3 } & \\ \text { Ca } & \text { BMM } & \text { M-SA } & \text { Mal } \\ \text { H } & \text { BMM, VS y PI } & \text { M-SA } & \text { Mal } \\ & & & \\ \text { L } & \text { BMM } & \text { E-DByEVT } & \\ \text { FE } & \text { BMM } & \text { M2 } & \end{array}$

Ca BMM, BPQ M3 Dis

FE BMM M

FE BMM M

P BMM M-An-SA Mal

H BMM M-An-SA Mal

$\mathrm{H} \quad \mathrm{BMM} \quad \mathrm{COS}$

T $\quad$ BMM $\quad$ M-SA

FE/FC BMM, BPQ M-CA 2:V

$\mathrm{FE} / \mathrm{FC} \quad \mathrm{BMM} \quad \mathrm{M} 2$

T VS y PI M2

H $\quad$ BMM $\quad$ M2 $\quad$ Mal

T VS y PI $\quad M \quad$ Mal

FE BMM, BPQ M3 $2: \mathrm{Br}$

FE $\quad$ BMM $\quad$ M1

P $\quad$ BPQ Cos

BMM

M-An-SA

Mal

\section{BMM}

E-DByEVT

Mal

$\mathrm{H}$

$\mathrm{T}$

$\mathrm{H}$

$\mathrm{H}$

FC

$\mathrm{H}$

$\mathrm{H}$

$\mathrm{H}$

FC

$B M M$

BMM, BPQ

E-EVT

Mal

\section{BMM}

M1

BMM

E-EVT

BMM

E-DByEVT

M2

BMM

E-CPyDB

BMM

M-SA

BMM

M1

BMM

M2

$\mathrm{H}$ BMM

M1

BMM

M2

FE BMM

E-DB

Mal

$\mathrm{H} \quad \mathrm{BMM}$

E-DByEVT

Mal

E-I 
Apéndice: Continuación

No. Familia/ especie

185 Marina gracillima (S.Watson) Barneby

186 Medicago polymorpha L.

187 Phaseolus pauciflorus Sessé \& Moc. ex G.Don

188 Phaseolus pedicellatus Benth.

189 Phaseolus vulgaris L.

190 Trifolium amabile Kunth Fagaceae

191 Quercus acutifolia Née

192 Quercus candicans Née

193 Quercus castanea Née

194 Quercus crassipes Humb. \& Bonpl.

195 Quercus glabrescens Benth.

196 Quercus glaucoides M.Martens \& Galeotti

197 Quercus laeta Liebm.

198 Quercus laurina Humb. \& Bonpl.

199 Quercus magnoliifolia Née

200 Quercus rugosa Née

201 Quercus scytophylla Liebm.

Flacourtiaceae

202 Xylosma flexuosa (Kunth) Hemsl.

Garryaceae

203 Garrya laurifolia Hartw. ex Benth.

Geraniaceae

204 Geranium seemannii Peyr.

Guttiferae

205 Hypericum philonotis Schltdl. \& Cham.

Hydrophyllaceae

206 Phacelia heterophylla Pursh

207 Phacelia platycarpa (Cav.) Spreng.

208 Wigandia urens (Ruiz \& Pav.) Kunth Lamiaceae

209 Hyptis mutabilis (Rich.) Briq.

210 Lepechinia caulescens (Ortega) Epling

211 Lepechinia nelsonii (Fernald) Epling

212 Marrubium vulgare $\mathrm{L}$.

213 Prunella vulgaris L.

214 Salvia elegans Vahl

215 Salvia fulgens Cav.

216 Salvia gesneriifolia Lindl. ex Lem

217 Salvia gracilis Benth.

218 Salvia helianthemifolia Benth.

219 Salvia lavanduloides Kunth

220 Salvia mexicana Sessé \& Moc.

221 Salvia mocinoi Benth.

222 Salvia polystachia Cav.

223 Salvia purpurea Cav.

224 Salvia sessei Benth.

225 Salvia tiliifolia Vahl

226 Scutellaria coerulea Moc. \& Sessé ex Benth.

FB Vegetación Distribución

Flora Categoría sinantrópica de riesgo

$\mathrm{H}$

$\mathrm{T}$

$\mathrm{Cr}$

$\mathrm{H}$

$\mathrm{H}$

$\mathrm{H}$

H

FE

FE

FE

FE

FE

$\mathrm{FE}$

FE

$\mathrm{FE}$

FE

FE

FE

FE

FE

$\mathrm{H}$

$\mathrm{T}$

$\mathrm{H}$

$\mathrm{H}$

FC

$\mathrm{H}$

$\mathrm{H}$

$\mathrm{Ca}$

$\mathrm{H}$

$\mathrm{H}$

$\mathrm{Ca}$

FC

FC

$\mathrm{H}$

$\mathrm{H}$

$\mathrm{H}$

$\mathrm{H}$

$\mathrm{Ca}$

$\mathrm{H}$

Ca

FC

$\mathrm{T}$

$\mathrm{H}$

BMM
VS y PI
BMM
BMM
BMM
BPQ

\section{E-SMOc}

Cos

M2

E-DByEVT

M-CA

M-CA

Mal

Int

$\begin{array}{ll}\text { BMM } & M 2 \\ \text { BMM } & \text { M2 }\end{array}$

BMM, BPQ

BMM

BMM

BMM

BMM

BMM

BMM

BPQ

$B M M, B P Q$

$\mathrm{M} 2$

$$
\text { E-EVT }
$$

E-DByEVT

M1

E-SMOcyEVT

E-EVT

$M$

M1

M

$$
\text { BMM }
$$

M3

BPQ

M-CA

BPQ

M2

Mal

BMM

M2

VS y PI
BMM
BPQ
BMM
BMM
BMM
VS y PI
BMM
BMM
BMM
BMM
BPQ
BMM
BPQ, VS y PI
BMM
BMM
BMM, BPQ
BMM
BMM
BMM
BMM

NA-M

M2

M-SA

Mal

M-SA

M-CA

M2

E-DB

Cos

NA-M-CA

$M$

E-EVT

E-EVT

M-CA

$M$

M2

$M$

$\mathrm{M} 2$

M-CA

$\mathrm{M} 2$

$M$

M-SA

M2 
Apéndice: Continuación

\section{No. Familia/ especie}

227 Stachys agraria Schltdl. \& Cham.

228 Stachys eriantha Benth.

Lauraceae

229 Nectandra salicifolia (Kunth) Nees Lentibulariaceae

230 Pinguicula moranensis Kunth Linaceae

231 Linum orizabae Planch. Loganiaceae

232 Buddleja parviflora Kunth

233 Buddleja sessiliflora Kunth Loranthaceae

234 Phoradendron velutinum (DC.) Oliv.

235 Psittacanthus calyculatus (DC.) G.Don Lythraceae

236 Cuphea aequipetala Cav.

237 Cuphea jorullensis Kunth Malvaceae

238 Anoda cristata (L.) Schltdl.

239 Sida rhombifolia L. Melastomataceae

240 Monochaetum calcaratum (DC.) Triana Menispermaceae

241 Cissampelos pareira L. Myrsinaceae

242 Myrsine juergensenii (Mez) Ricketson \& Pipoly

243 Parathesis melanosticta (Schltdl.) Hemsl. Oleaceae

244 Fraxinus uhdei (Wenz.) Lingelsh. Onagraceae

245 Fuchsia arborescens Sims

246 Fuchsia thymifolia Kunth

247 Gaura coccinea Pursh.

248 Lopezia miniata DC.

249 Lopezia racemosa Cav.

250 Oenothera epilobiifolia Kunth

251 Oenothera pubescens Willd. ex Spreng.

252 Oenothera rosea Aiton

\section{Oxalidaceae}

253 Oxalis alpina Rose

254 Oxalis corniculata L.

255 Oxalis tetraphylla Cav.

\section{Passifloraceae}

256 Passiflora pavonis Mast. Phytolaccaceae

257 Phytolacca icosandra L. Piperaceae

258 Peperomia campylotropa A.W.Hill

259 Peperomia galioides Kunth

\section{FB \\ Vegetación Distribución} sinantrópica de riesgo

\begin{tabular}{|c|c|c|}
\hline $\mathrm{H}$ & BMM & M3 \\
\hline $\mathrm{H}$ & BMM & E-EVT \\
\hline FE & $\mathrm{BMM}$ & M2 \\
\hline $\mathrm{Ca}$ & BMM & M2 \\
\hline $\mathrm{H}$ & BMM & $M$ \\
\hline $\mathrm{FC}$ & $\mathrm{BMM}$ & $M$ \\
\hline FC & BMM & M1 \\
\hline HP & $\mathrm{BMM}, \mathrm{BPQ}$ & M2 \\
\hline HP & BMM & M-SA \\
\hline $\mathrm{H}$ & BMM & M2 \\
\hline $\mathrm{H}$ & BMM & -SMOcyEVT \\
\hline $\mathrm{Ca}$ & BMM & M-SA \\
\hline $\mathrm{Ca}$ & BMM, VS y PI & Cos \\
\hline FC & BMM & $M$ \\
\hline $\mathrm{H}$ & BMM & Cos \\
\hline FC & BMM & $\mathrm{M}-\mathrm{CA}$ \\
\hline FC & BMM & M \\
\hline FE & BMM & $M$ \\
\hline $\mathrm{FE}$ & BMM & $\mathrm{M}-\mathrm{CA}$ \\
\hline FC & $\mathrm{BPQ}$ & M2 \\
\hline $\mathrm{H}$ & BMM & NA-M \\
\hline $\mathrm{Ca}$ & BMM & $\mathrm{M}-\mathrm{CA}$ \\
\hline $\mathrm{H}$ & $\mathrm{BMM}, \mathrm{BPQ}$ & M2 \\
\hline $\mathrm{H}$ & BMM & M-SA \\
\hline $\mathrm{H}$ & BMM & M-SA \\
\hline $\mathrm{H}$ & BMM & M-SA \\
\hline $\mathrm{Cr}$ & BMM & M3 \\
\hline $\mathrm{Cr}$ & BMM, BPQ, VS y PI & 기 M-CA \\
\hline $\mathrm{Cr}$ & BMM & M-An-CA \\
\hline $\mathrm{H}$ & BMM & M2 \\
\hline $\mathrm{Ca}$ & $\mathrm{BPQ}$ & M-SA \\
\hline $\mathrm{Cr}$ & $\mathrm{BPQ}$ & M2 \\
\hline $\mathrm{H}$ & BMM, BPQ & M-An-SA \\
\hline
\end{tabular}


Apéndice: Continuación

No. Familia/ especie

260 Peperomia quadrifolia (L.) Kunth

261 Piper aff. amalago L.

Plantaginaceae

262 Plantago australis Lam. ssp. hirtella (Kunth) Rahn

263 Plantago major L.

Polemoniaceae

264 Loeselia glandulosa (Cav.) G.Don Polygalaceae

265 Monnina ciliolata Sessé \& Moc. ex DC.

266 Polygala gracillima S.Watson Polygonaceae

267 Polygonum hydropiperoides Michx.

268 Polygonum mexicanum Small

269 Polygonum punctatum Elliott Primulaceae

270 Anagallis arvensis L.

Ranunculaceae

271 Clematis dioica L.

272 Ranunculus dichotomus Moc. \& Sessé ex DC.

273 Ranunculus petiolaris Kunth ex DC.

274 Thalictrum strigillosum Hemsl. Resedaceae

275 Reseda luteola L. Rosaceae

276 Acaena elongata L.

277 Alchemilla pringlei (Rydb.) Fedde

278 Alchemilla procumbens Rose

279 Crataegus mexicana Moc. \& Sessé ex DC.

280 Crataegus pubescens C.Presl

281 Prunus serotina Ehrh.

282 Rubus liebmannii Focke Rubiaceae

283 Bouvardia ternifolia (Cav.) Schltdl.

284 Crusea coccinea DC.

285 Didymaea floribunda Rzed.

286 Galium uncinulatum DC. Sabiaceae

287 Meliosma dentata Urb. Salicaceae

288 Salix bonplandiana Kunth Saxifragaceae

289 Ribes affine Kunth Scrophulariaceae

290 Mimulus glabratus Kunth

291 Penstemon campanulatus (Cav.) Willd.

292 Verbascum virgatum Stokes Solanaceae

293 Cestrum nitidum M.Martens \& Galeotti 294 Cestrum roseum Kunth
FB Vegetación Distribución $\begin{array}{cc}\text { Flora } & \text { Categoría } \\ \text { sinantrópica } & \text { de riesgo }\end{array}$

$\begin{array}{cccc}\text { E } & \text { BMM } & \text { M-An-SA } & \text { Mal } \\ \text { FC } & \text { BMM } & \text { M-SA } & \\ & & & \\ \text { H } & \text { BMM } & \text { M-SA } & \text { Mal } \\ \text { H } & \text { VS y PI } & \text { M-SA } & \text { Int }\end{array}$

H $\quad$ BMM $\quad$ A

FC BMM E-DByEVT

$\begin{array}{lll}\mathrm{H} & \mathrm{BMM}\end{array}$

$\mathrm{H} \quad$ VS y PI $\quad \mathrm{A}$

T $\quad$ BMM $\quad$ M1

$\mathrm{Cr} \quad$ VS y PI

A

Mal

1

Cos

Mal

$\mathrm{T}$

VS y PI

Cos

Int

L

$\mathrm{Cr}$

$\mathrm{Cr}$

$\mathrm{H}$

\section{BMM}

VS y PI

M-An-SA

Mal

M-SA

VS y $\mathrm{PI}$

M-SA

Mal

$M$

$\mathrm{T}$

VS y PI

Cos

Int

Ca

BMM

M-SA

$\mathrm{H}$

BMM

$M 2$

$M-S A$

Dis

Ca BMM

$M-S A$

BMM

$M 2$

M3

M

FC BMM

Mal

FE

BMM

M1

$\mathrm{M} 2$

$M$

$\mathrm{H}$

VS y PI

VS y PI

BMM

M-CA

FE

BMM

M2

FE

BMM

M3

FC

BMM, BPQ

E-EVT

$\mathrm{H}$

$\mathrm{H}$

$$
\text { BMM }
$$

BMM

BMM

A
M2
Cos

Mal

$\mathrm{T}$

BMM

FC

BMM

$M$

M

Dis 
Apéndice: Continuación

\section{No. Familia/ especie}

FB

295 Lycianthes peduncularis (Schltdl.) Bitter

296 Physalis coztomatl Moc. \& Sessé ex Dunal

297 Physalis philadelphica Lam.

298 Physalis viscosa L.

299 Solandra nitida Zucc.

300 Solanum appendiculatum Humb. \& Bonpl. ex Dunal

301 Solanum cervantesii Lag.

302 Solanum marginatum L.f.

303 Solanum nigrescens M.Martens \& Galeotti Styracaceae

304 Styrax argenteus C.Presl var. ramirezii (Greenm.) Gonsoulin

\section{Symplocaceae}

305 Symplocos citrea La Llave \& Lex. Theaceae

306 Cleyera integrifolia (Benth.) Choisy

307 Ternstroemia lineata DC. ssp. lineata

308 Ternstroemia sylvatica Cham. \& Schltdl. Tiliaceae

309 Tilia americana L. var. mexicana (Schltdl.) Hardin

310 Triumfetta columnaris Hochr. Valerianaceae

311 Valeriana clematitis Kunth

312 Valeriana sorbifolia Kunth Verbenaceae

313 Citharexylum affine D.Don

314 Lantana velutina M.Martens \& Galeotti

315 Lippia umbellata Cav.

316 Priva grandiflora (Ortega) Moldenke

317 Verbena bipinnatifida Nutt.

318 Verbena carolina L. Violaceae

319 Viola humilis Kunth Vitaceae

320 Vitis tiliifolia Humb. \& Bonpl. ex Schult. LILIOPSIDA Agavaceae

321 Manfreda pringlei Rose

322 Polianthes geminiflora (Lex.) Rose Alstroemeriaceae

323 Bomarea hirtella (Kunth) Herb. Anthericaceae

324 Echeandia durangensis (Greenm.) Cruden Bromeliaceae

325 Tillandsia dasyliriifolia Baker

326 Tillandsia dugesii Baker

327 Tillandsia prodigiosa (Lem.) Baker

328 Tillandsia usneoides (L.) L.

$\begin{array}{cccc}\text { H } & \text { BPQ } & \text { E-DByEVT } & \\ \text { Ca } & \text { BMM } & \text { E-DByEVT } & \text { Mal } \\ \text { T } & \text { VS y PI } & \text { M-An-CA } & \text { Mal } \\ \text { Ca } & \text { BMM } & \text { M-SA } & \\ \text { L } & \text { BMM } & M & \\ \text { Ca } & \text { BMM, BPQ } & \text { M2 } & \\ \text { FC } & \text { BMM, VS y PI } & \text { M2 } & \text { Int } \\ \text { FC } & \text { VS y PI } & \text { Cos } & \text { Mal } \\ \text { Ca } & \text { BMM } & \text { M-SA } & \\ & & & \\ \text { FE } & \text { BMM } & \text { M-CA } & \end{array}$

FE

BMM

E-DByEVT

$1: \operatorname{Pr}$

FE

BMM

E-SMOcyDB

BMM

$\mathrm{E}-\mathrm{CP}$

FE

BMM

E-SMOcyDB

FE

BPQ

$M$

M

$\mathrm{Cr}$

BPQ

BMM

M2

$\mathrm{T}$

BMM

$M-S A$

FC

BMM

$M$

FC BMM

NA-M

BMM

M2

$M$

M3

M2

Dis

BMM

BMM

BMM

E-DByEVT

L $\quad$ BMM, BPQ

M-An-SA

$\mathrm{H}$

$\mathrm{Cr}$

BMM

M2

M

$\mathrm{H}$

BMM

M

Dis

$\mathrm{Cr}$

BMM

M

E

BMM

M2

BMM, BPQ

BMM

$M$

BMM 
Apéndice: Continuación

\section{No. Familia/ especie}

FB Vegetación Distribución

\section{sinantrópica}

Categoría de riesgo

\section{Commelinaceae}

329 Commelina coelestis Willd.

330 Commelina diffusa Burm.f.

331 Commelina tuberosa L.

332 Cymbispatha commelinoides (Schult. \& Schult.f) Pichon

333 Gibasis linearis (Benth.) Rohweder

334 Gibasis pulchella (Kunth.) Raf.

335 Tripogandra amplexicaulis (Klotzsch ex C.B.Clarke) Woodson

\section{Convallariaceae}

336 Maianthemum scilloideum (M.Martens \& Galeotti) LaFrankie

337 Smilacina flexuosa Bertol. Cyperaceae

338 Cyperus aggregatus (Willd.) Endl.

339 Cyperus hermaphroditus (Jacq.) Standl.

340 Cyperus niger Ruiz \& Pav.

341 Cyperus odoratus L.

342 Cyperus semiochraceus Boeckeler

343 Cyperus spectabilis Link

Dioscoreaceae

344 Dioscorea convolvulacea Schltdl. \& Cham.

345 Dioscorea urceolata Uline

Hydrocharitaceae

346 Egeria densa Planch.

Hypoxidaceae

347 Hypoxis mexicana Schult. \& Schult.f. Iridaceae

348 Sisyrinchium scabrum Cham. \& Schltdl. Juncaceae

349 Luzula racemosa Desv. Orchidaceae

350 Corallorhiza wisteriana Conrad

351 Epidendrum anisatum La Llave \& Lex

352 Erycina hyalinobulbon (La Llave \& Lex.) N.H.Williams \& M.W.Chase

353 Habenaria clypeata Lindl.

354 Habenaria guadalajarana S.Watson

355 Habenaria jaliscana S.Watson

356 Hintonella mexicana Ames

357 Isochilus bracteatus (La Llave \& Lex.) Salazar \& Soto Arenas ex Espejo \& López-Ferrari

358 Maxillaria cucullata Lindl.

359 Maxillaria lexarzana Soto Arenas \& F.Chiang

360 Neottia micrantha La Llave \& Lex.

361 Oncidium reichenheimii (Linden \& Rchb.f.) Garay \& Stacy

362 Rhynchostele cervantesii (La Llave \& Lex.) Soto Arenas \& Salazar

$\begin{array}{cccc}\mathrm{Cr} & \text { BMM } & \text { M2 } & \text { Mal } \\ \mathrm{Cr} & \text { BMM } & \text { M-SA } & \text { Mal } \\ \mathrm{Cr} & \text { BMM } & \text { M2 } & \text { Mal } \\ \mathrm{Cr} & \text { BMM } & \text { M-CA } & \text { Mal } \\ \mathrm{Cr} & \text { BMM } & \text { E-SMOcyEVT } & \\ \mathrm{Cr} & \text { BMM } & \text { E-EVT } & \\ \mathrm{T} & \text { BMM } & \text { M2 } & \text { Dis }\end{array}$

$\mathrm{Cr}$

BMM

$\mathrm{M} 2$

$\mathrm{H}$

BMM

M2

$\mathrm{H}$

$\mathrm{H}$

$\mathrm{H}$

$\mathrm{H}$

$\mathrm{H}$

$\mathrm{H}$

\section{BMM}

BMM

BMM

BMM

$\checkmark S$ y $\mathrm{PI}$

VS y PI

M-An-SA

A

A

Cos

Mal

M-CA

M-SA

Mal

\section{$\mathrm{H}$}

$\mathrm{Ca}$

BPQ

VS y PI

M-CA

E-I

$\mathrm{Hi}$

VA

Cos

Int

$\mathrm{Cr}$

$$
\text { BMM }
$$

M1

Dis

$\mathrm{Cr}$

BMM

M2

Mal

$\mathrm{H}$

BMM

M-SA

P

E

E

BMM

E-EVT

\section{BMM}

E-SMOcyDB

BMM

E-DByEVT

Cr BMM

M-CA

$\mathrm{Cr}$ BMM

$M-S A$

$M$

$\mathrm{Cr}$ BMM

E BMM

E BMM

E-DByEVT

E-I

Cr BMM

M-CA

E BMM

$\mathrm{Cr} \quad \mathrm{BMM}$

E BMM

\section{E-I}

M-CA

E-DB

E

BMM

E-CP 
Apéndice: Continuación

\section{No. Familia/ especie}

FB Vegetación Distribución sinantrópica
363 Stelis villosa (Knowles \& Westc.) Pridgeon \& M.W.Chase E

364 Tamayorkis ehrenbergii (Rchb.f.) R.González \& Szlach. Cr

365 Trichocentrum pachyphyllum (Hook.) R.Jiménez \& Carnevali

\section{Poaceae}

366 Aristida schiedeana Trin. \& Rupr.

367 Aristida ternipes Cav.

368 Bouteloua curtipendula (Michx.) Torr. var. tenuis Gould \& Kapadia

369 Briza minor L.

370 Bromus carinatus Hook. \& Arn.

371 Eragrostis mexicana (Hornem.) Link

372 Festuca amplissima Rupr.

373 Muhlenbergia capillaris (Lam.) Trin.

374 Muhlenbergia robusta (E.Fourn.) Hitchc.

375 Oplismenus burmannii (Retz.) P. Beauv.

376 Panicum sphaerocarpon Elliott

377 Paspalum notatum Flüggé

378 Paspalum squamulatum E.Fourn.

379 Pennisetum clandestinum Hochst. ex Chiov.

380 Pennisetum crinitum (Kunth) Spreng.

381 Peyritschia deyeuxioides (Kunth) Finot

382 Poa annua L.

383 Polypogon elongatus Kunth

384 Setaria parviflora (Poir.) Kerguélen

385 Trachypogon spicatus (L.f.) Kuntze

386 Trisetum virletii E.Fourn.

387 Zeugites americana Willd.

var. pringlei (Scribn.) McVaugh

\section{Smilacaceae}

388 Smilax moranensis M. Martens \& Galeotti

389 Smilax pringlei Greenm.

Typhaceae

390 Typha latifolia L.

Zannichelliaceae

391 Zannichellia palustris L.

$\begin{array}{lll}\text { E } & \text { BMM } & \text { M2 } \\ \text { Cr } & \text { BMM } & \text { M3 } \\ \text { E } & \text { BMM } & \text { M2 }\end{array}$

\section{M2}

M3

$\begin{array}{llll}H & B P Q & M 2 & \text { Mal }\end{array}$

H BPQ M-SA

$\mathrm{H} \quad \mathrm{BPQ} \quad \mathrm{A} \quad \mathrm{Mal}$

T BMM $\quad$ Cos Int

$\mathrm{H} \quad \mathrm{VS}$ y PI M-CA Mal

T VS y PI M-SA Mal

$\mathrm{H} \quad \mathrm{BPQ} \quad \mathrm{M}-\mathrm{CA}$

$\begin{array}{llll}H & B P Q & M 1 & \text { Mal }\end{array}$

$\mathrm{H} \quad \mathrm{BPQ} \quad \mathrm{M} 2$

$\begin{array}{lll}\mathrm{T} & \mathrm{BPQ} & \mathrm{COS}\end{array}$

$\mathrm{H} \quad \mathrm{BPQ} \quad \mathrm{M}-\mathrm{SA}$

H BPQ M-An-SA Mal

$\mathrm{H} \quad \mathrm{BPQ} \quad \mathrm{M}-\mathrm{CA}$ Dis

$\mathrm{H} \quad \mathrm{VS}$ y PI $\mathrm{Cos}$ Int

H BPQ M Mal

H BMM M-SA

$\mathrm{H} \quad \mathrm{BMM} \quad \mathrm{CoS}$ Int

$\mathrm{H}$ BPQ M-SA Mal

$\mathrm{H}$ BPQ Cos Dis

$\mathrm{H}$ BPQ M-An-SA

Ca BPQ E-EVT

$\begin{array}{lll}\mathrm{H} & \mathrm{BMM} & \mathrm{M} 2\end{array}$

$\begin{array}{cccc}\text { L } & \text { BMM, BPQ } & \text { M } & \\ \text { L } & \text { BMM } & M & \\ \mathrm{Hi} & \text { VA } & \text { Cos } & \text { Mal } \\ \mathrm{Hi} & \text { VA } & \text { Cos } & \text { Mal }\end{array}$

